\title{
Asymptotic and Exact Expansions of Heat Traces
}

\author{
Michał Eckstein ${ }^{1} \cdot$ Artur Zając ${ }^{2}$
}

Received: 16 March 2015 / Accepted: 8 September 2015 / Published online: 20 October 2015

(C) The Author(s) 2015. This article is published with open access at Springerlink.com

\begin{abstract}
We study heat traces associated with positive unbounded operators with compact inverses. With the help of the inverse Mellin transform we derive necessary conditions for the existence of a short time asymptotic expansion. The conditions are formulated in terms of the meromorphic extension of the associated spectral zetafunctions and proven to be verified for a large class of operators. We also address the problem of convergence of the obtained asymptotic expansions. General results are illustrated with a number of explicit examples.
\end{abstract}

Keywords Heat traces - Asymptotic expansions - Spectral theory $\cdot$ Zeta-functions · General Dirichlet series

Mathematics Subject Classification (2010) Primary: 11M36 · Secondary: $30 \mathrm{~B} 50 \cdot 35 \mathrm{~K} 08 \cdot 41 \mathrm{~A} 60 \cdot 47 \mathrm{~B} 15 \cdot 81 \mathrm{Q} 10$

Michał Eckstein

michal@eckstein.pl

Artur Zając

artur.zajac@uj.edu.pl

1 Faculty of Physics, Astronomy and Applied Computer Science, Jagiellonian University, ul. prof. Stanisława Łojasiewicza 11, 30-348 Kraków, Poland

2 Faculty of Mathematics and Computer Science, Jagiellonian University, ul. prof. Stanisława Łojasiewicza 6, 30-348 Kraków, Poland 


\section{Introduction}

Given a positive, possibly unbounded, operator $P$ with a compact resolvent, acting on a separable infinite-dimensional Hilbert space $\mathcal{H}$ one can define the associated heat operator $\mathrm{e}^{-t P}$ for $t>0$. The latter, under some mild conditions on $P$, is trace class for any $t>0$. It turns out that a close inspection of the function $t \mapsto \operatorname{Tr} \mathrm{e}^{-t P}$, reveals a lot of information of geometrical nature. For instance, if $P$ is a differential operator of Laplace type defined on a closed Riemannian manifold then the classical results [40] show that there exists an asymptotic expansion of the form

$$
\operatorname{Tr} \mathrm{e}^{-t P} \underset{t \downarrow 0}{\sim} \sum_{k \geqslant 0} a_{k}(P) t^{(k-d) / 2},
$$

where $d$ is the dimension of the manifold and $a_{k}$ 's - called Seeley-DeWitt coefficients - are given by the integrals over the manifold of some geometrical invariants. Moreover, the coefficients $a_{k}$ can be expressed as

$$
a_{k}(P)=\operatorname{Res}_{s=(d-k) / 2} \Gamma(s) \zeta_{P}(s),
$$

where $\zeta_{P}(s):=\operatorname{Tr} P^{-s}$ is the (meromorphic extension of the) spectral zeta-function associated with $P$.

The existence of an asymptotic expansion of $\operatorname{Tr}^{-t P}$ was proven for $P$ being a classical positive elliptic pseudodifferential operator of order $m \in \mathbb{N}$ (see [39] and references therein). In this case,

$$
\operatorname{Tr} \mathrm{e}^{-t P} \underset{t \downarrow 0}{\sim} \sum_{k=0}^{\infty} a_{k}(P) t^{(-d+k) / m}+\sum_{l=1}^{\infty} b_{l}(P) t^{l} \log t
$$

and

$$
a_{k}(P)=\operatorname{Res}_{s=(d-k) / m} \Gamma(s) \zeta_{P}(s), \quad b_{l}(P)=-\operatorname{Res}_{s=-l}(s+l) \Gamma(s) \zeta_{P}(s) .
$$

In fact, this result can be extended to certain classes of non-classical pseudodifferential operators. In [53] for instance, the heat traces of pseudodifferential operators with log-polyhomogeneous symbols have been studied.

With the development of noncommutative geometry [16], the need came to investigate heat traces associated with positive functions of Dirac operators in the framework of spectral triples. Unfortunately, there is no analogue of the formula (1) for a general spectral triple [72]. In fact, the existence of an asymptotic expansion of the heat trace is assumed whenever needed in applications (see for instance [19, Section 11], [21, Section 2.1]) and has been proven rigorously only for a few specific examples $[29,37,71]$. Whence the results of $[37,71]$ essentially used the methods of pseudodifferential calculus, the casus of the standard Podleś sphere [29] required completely different tools (see Section 4.2).

We note that the interplay between heat traces and spectral zeta-functions has been investigated in a very general framework of von Neumann algebras by a number of authors [7, 16, 54, 67]. However, the efforts of the latter focused on the leading behaviour of heat traces and its consequences for measurability. 
The purpose of this paper is to study heat traces associated with general densely defined positive operators $P$ with compact inverses. In particular, we formulate sufficient conditions for the existence of a small $t$ asymptotic expansion of $\operatorname{Tr}^{-t P}$. Having an asymptotic expansion at hand, a natural question one may pose is that of its convergence. This issue has not been studied in full generality, even in the context of $P$ being a differential operator (see however $[29,46]$ ). We show how the conditions on $P$ shall be refined in order to get an exact formula for the heat trace valid on some open interval $(0, T)$. The motivation behind our work comes from noncommutative geometry, but the framework of the studies is even wider.

The heat trace methods have multifarious applications in theoretical physics (see [70] for a review). They are in common use in quantum field theory [2, 5, 30, 36], also in its noncommutative version [38]. In general, one only disposes of an asymptotic expansion of the heat trace as $t \downarrow 0$. This implies that the field-theoretic calculations performed with the help of this method are only perturbative. Needless to say that a control on the convergence of a perturbative expansion is of crucial importance.

In noncommutative geometry, the heat trace is the cornerstone of bosonic spectral action computations [9, 19, 46-48]. The large energies expansion of the latter is based on the asymptotic expansion of the heat trace associated with the relevant Dirac operator. Recently, also the exact computations of the spectral action gained interest $[10,28,29,57,58,61,68]$ due to their possible application to the study of cosmic topology.

The plan of the paper presents itself as follows: In Section 2 we recollect some basic notions on spectral functions associated with positive operators. Then, in Section 3 we discuss in details the interplay between the meromorphic extension of the spectral zeta-function $\zeta_{P}$ and the asymptotic expansion of the associated heat trace $\operatorname{Tr} \mathrm{e}^{-t P}$, by gathering results on general Dirichlet series [43, 44] and the Mellin transform [35, 63]. Moreover, we present a set of sufficient assumptions on $P$ so that the associated heat trace is controlled for $t$ in some non-empty open interval. Section 4 illustrates the general theorems with various special cases and examples coming from Dirac-type operators on both classical manifolds and noncommutative spaces. We end with an outlook on the possible generalisations and applications of our results. We also discuss the limitations of the method and compare its usefulness with the Tauberian theorems commonly used in this domain.

\section{Preliminaries}

\subsection{Notations}

Let us first fix some notations:

- $\mathbb{N}$ denotes the non-negative integers, $\mathbb{N}^{+}$the positive ones, $\mathbb{Z}^{*}$ stands for the non-zero integers and $\mathbb{R}^{+}$for positive reals.

- Unless stated otherwise, $t$ will always denote a positive parameter and $s$ a complex one.

- $f(s) \approx g(s)$ means that $\lim _{|s| \rightarrow \infty} f(s) / g(s)=1$. 
- $\quad f(x)=\mathcal{O}_{x \rightarrow x_{0}}(g(x))$ means that $\limsup _{x \rightarrow x_{0}}|f(x) / g(x)|<\infty$, for $x, x_{0} \in$ $\mathbb{R} \cup\{ \pm \infty\}$. The notation $f(x)=\mathcal{O}_{x_{0}}(g(x))$ will be used when the variable is obvious.

- $f(x)=\mathcal{O}_{x \rightarrow x_{0}}(g(x))$ means that $\limsup _{x \rightarrow x_{0}}|f(x) / g(x)|=0$, for $x, x_{0} \in$ $\mathbb{R} \cup\{ \pm \infty\}$. The notation $f(x)=\mathcal{O}_{x_{0}}(g(x))$ will be used when the variable is obvious.

- $f(t) \underset{t \downarrow 0}{\sim} \sum_{n} \phi_{n}(t)$ denotes an asymptotic expansion (see Definition 4) of $f$ as $t$ tends to 0 from above.

\subsection{Heat Traces}

Let $P$ denote an unbounded operator on a Hilbert space. Throughout the paper we will assume the following on $P$ :

General Assumption $P$ is a positive densely defined operator with a compact inverse, acting on a separable infinite-dimensional Hilbert space.

Such a strong assumption allows us, among other things, to use freely the spectral theorem for $P$. As a consequence, the spectrum $\sigma(P)$ is a discrete subset of $\mathbb{R}$, which can be ordered into a sequence increasing to infinity

$$
\sigma(P)=\left(\lambda_{n}\right)_{n=0}^{\infty}, \quad 0<\lambda_{0}<\lambda_{1}<\ldots, \quad \lim _{n \rightarrow \infty} \lambda_{n}=\infty .
$$

We will denote the multiplicity of the eigenvalue $\lambda_{n} \in \sigma(P)$ by $M_{n}$. For further purposes, we also define the spectral growth function as

$$
N(\lambda):=\sum_{\left\{n: \lambda_{n} \leqslant \lambda\right\}} M_{n} .
$$

Now we define the main object of our interest:

Definition 1 The heat trace of the operator $P$ is the function $\operatorname{htr}_{P}: \mathbb{R}^{+} \rightarrow \mathbb{R}^{+}$, defined as

$$
\operatorname{htr}_{P}(t):=\operatorname{Tr} \mathrm{e}^{-t P} .
$$

We say that the heat trace is well-defined if $\mathrm{e}^{-t P}$ is a trace class operator for any $t>0$.

Using (2) the heat trace can be written as

$$
\operatorname{htr}_{P}(t)=\sum_{n=0}^{\infty} M_{n} \mathrm{e}^{-t \lambda_{n}} .
$$

The sum is of the form of a general Dirichlet series, which is defined as

$$
\sum_{n=0}^{\infty} a_{n} \mathrm{e}^{-s b_{n}},
$$

for $s$ in some (possibly empty) subset of $\mathbb{C}, a_{n} \in \mathbb{C}$ and $\left(b_{n}\right)_{n=0}^{\infty}$ a sequence of real numbers increasing to infinity. Throughout this paper we will meet only examples 
with $a_{n}$ real and non-negative for $n \in \mathbb{N}$ (usually we will simply have $a_{n}=M_{n}$ ). In such a case the convergence of (5) is governed by the following theorem:

Theorem 1 ([44], Theorem 7 with the footnote) If $a_{n}>0$ for $n \in \mathbb{N}$ and $\sum_{n=0}^{\infty} a_{n}=\infty$ then the general Dirichlet series (5) converges for $\mathfrak{R}(s)>L$ and diverges for $\Re(s)<L$, where $L$ is given by

$$
L=\limsup _{n \rightarrow \infty} b_{n}^{-1} \log \left(a_{0}+\ldots+a_{n}\right),
$$

and $L \geqslant 0$.

We will call such $L$ the abscissa of convergence of the general Dirichlet series (5). The inequality $L \geqslant 0$ follows from the fact that for $s=0$ the series (5) is equal to $\sum_{n=0}^{\infty} a_{n}$, which is divergent. Note that the abscissa can be infinite, what means that the series is nowhere convergent.

Proposition 1 The heat trace of the operator $P$ is well-defined:

1. if and only if $N\left(\lambda_{n}\right)=\mathcal{O}_{n \rightarrow \infty}\left(\mathrm{e}^{\epsilon \lambda_{n}}\right)$ for any positive $\epsilon$;

2. if there exist $\alpha \geqslant 0$ such that $M_{n}=\mathcal{O}_{\infty}\left(n^{\alpha}\right)$ and $\log n=\mathcal{O}_{\infty}\left(\lambda_{n}\right)$ (i.e. $\lambda_{n}$ grow faster than $\log n)$.

Proof In order to have $\operatorname{htr}_{P}$ well-defined, we need the abscissa of convergence $L$ of the series (4) to be 0 . Taking $a_{n}=M_{n}, b_{n}=\lambda_{n}$ for all $n \in \mathbb{N}$ we see that $\sum_{n=0}^{\infty} a_{n}=\infty$ as $P$ has an infinite number of eigenvalues. Now, by Theorem 1 we get that $\operatorname{htr}_{P}$ is well-defined iff $L=\limsup _{n \rightarrow \infty} \lambda_{n}^{-1} \log N\left(\lambda_{n}\right)$ equals to 0 . This is equivalent to the statement that for any $\epsilon>0$ there exists $n_{0}$ such that for any $n \geqslant n_{0}$ we have $\lambda_{n}^{-1} \log N\left(\lambda_{n}\right) \leqslant \epsilon$ or, equivalently, $N\left(\lambda_{n}\right) \leqslant$ $\mathrm{e}^{\epsilon \lambda_{n}}$. This in turn is equivalent to $N\left(\lambda_{n}\right)=\mathcal{O}_{\infty}\left(\mathrm{e}^{\epsilon \lambda_{n}}\right)$ for any $\epsilon>0$ and thus 1 follows.

For statement 2 let us first note that since $M_{n}=\mathcal{O}_{\infty}\left(n^{\alpha}\right)$ for some $\alpha \geqslant 0$, then $N\left(\lambda_{n}\right)=\mathcal{O}_{\infty}\left(n^{\alpha+1}\right)$. Take a positive constant $C$ such that $N\left(\lambda_{n}\right) \leqslant C n^{\alpha+1}$ for $n \geqslant N$ for some $N \in \mathbb{N}$. Then,

$$
L=\limsup _{n \rightarrow \infty} \lambda_{n}^{-1} \log \left(N\left(\lambda_{n}\right)\right) \leqslant \limsup _{n \rightarrow \infty} \lambda_{n}^{-1} \log \left(C n^{\alpha+1}\right)=\limsup _{n \rightarrow \infty} \lambda_{n}^{-1}(\log C+(\alpha+1) \log n),
$$

what tends to 0 under the hypothesis of 2 .

Note that the condition in 2 is only sufficient: consider e.g. $\lambda_{n}=n^{2}$, and $M_{n}=2^{n}$ which satisfies the hypothesis of 1 but not the one of 2 .

To conclude this subsection, we remark that heat traces can be defined for operators bounded from below, possibly with a non-trivial kernel. However, the zetafunctions described below apply only to positive invertible operators (see, however, Section 3.3). 


\subsection{Spectral Zeta-functions}

Definition 2 The zeta-function associated with the operator $P$ is a complex function

$$
\mathbb{C} \supset \operatorname{Dom}\left(\zeta_{P}\right) \ni s \mapsto \zeta_{P}(s)=\operatorname{Tr} P^{-s} .
$$

We say that $\zeta_{P}$ is well-defined if $\operatorname{Dom}\left(\zeta_{P}\right)$ is non-empty.

Using the spectral theorem this can be written as

$$
\zeta_{P}(s)=\sum_{n=0}^{\infty} M_{n} \lambda_{n}^{-s}
$$

which is again a general Dirichlet series (5) with $a_{n}=M_{n}$ and $b_{n}=\log \lambda_{n}$. Using Theorem 1 we get

Proposition 2 The abscissa of convergence of the zeta-series (6) is given by

$$
L=\inf \left\{\alpha \in \mathbb{R}: N\left(\lambda_{n}\right)=\mathcal{O}_{n \rightarrow \infty}\left(\lambda_{n}^{\alpha}\right)\right\} .
$$

Note that it may happen that $L=+\infty$, which means that the zeta-function is not well-defined (e.g. when $\lambda_{n}=\log n, M_{n}=1$ for $n \geqslant 2$, then $N(\lambda) \approx \exp \lambda$ ).

Proof Take any $\alpha$ such that there exists a constant $c$ satisfying $N\left(\lambda_{n}\right) \leqslant c \lambda_{n}^{\alpha}$. Then by setting $a_{n}=M_{n}, b_{n}=\log \lambda_{n}$ we have by Theorem 1

$$
L=\limsup _{n \rightarrow \infty} \frac{\log N\left(\lambda_{n}\right)}{\log \lambda_{n}} \leqslant \limsup _{n \rightarrow \infty} \frac{\log \left(c \lambda_{n}^{\alpha}\right)}{\log \lambda_{n}}=\alpha .
$$

Thus $L \leqslant K$, where $K$ denotes the RHS of (7).

On the other hand, if we suppose that $L<K$ then there exists $\alpha$ such that $L<$ $\alpha<K$. Using (8) we can find such $n_{0} \in \mathbb{N}$ that $\log N\left(\lambda_{n}\right)\left(\log \lambda_{n}\right)^{-1}<\alpha$ for any $n \geqslant n_{0}$. Then we get that $N\left(\lambda_{n}\right)<\lambda_{n}^{\alpha}$ for $n \geqslant n_{0}$, which contradicts the assumption $\alpha<K$. Thus $L=K$ and the proposition is proved.

Proposition 3 If an operator $P$ is such that its zeta-function is well-defined with finite abscissa of convergence $L$, then its heat trace is also well-defined and

$$
\operatorname{htr}_{P}(t)=\mathcal{O}_{0}\left(t^{-\alpha}\right), \text { for all } \alpha>L .
$$

Proof Comparing Proposition 1 assertion 1. with Proposition 2 we get that $\operatorname{htr}_{P}$ is well-defined.

Now, let us take any $\alpha>L \geqslant 0$. Then, there exists a positive constant $C(\alpha)$ such that

$$
x^{\alpha} \mathrm{e}^{-x} \leqslant C(\alpha)
$$

for any $x>0$, as the function $x \mapsto x^{\alpha} \mathrm{e}^{-x}$ is bounded on $\mathbb{R}^{+} \cup\{0\}$ for any $\alpha \geqslant 0$. Therefore, for any $\alpha>L$ we have

$$
0 \leqslant t^{\alpha} \operatorname{htr}_{P}(t)=\sum_{n=0}^{\infty} M_{n} t^{\alpha} \mathrm{e}^{-t \lambda_{n}} \leqslant C(\alpha) \sum_{n=0}^{\infty} M_{n} \lambda_{n}^{-\alpha}=C(\alpha) \zeta_{P}(\alpha)<\infty .
$$


Hence, $\operatorname{htr}_{P}(t)=\mathcal{O}_{0}\left(t^{-\alpha}\right)$.

\subsection{Mellin Transform}

Definition 3 (see [63] for instance) The Mellin transform of a locally Lebesgue integrable function $f$ defined over $\mathbb{R}^{+}$is a complex function $\mathcal{M}[f]$ given by

$$
\mathcal{M}[f](s)=\int_{0}^{\infty} f(t) t^{s-1} d t .
$$

The inverse Mellin transform of a meromorphic function $g$, denoted by $\mathcal{M}^{-1}[g]$, reads

$$
\mathcal{M}^{-1}[g](t)=\frac{1}{2 \pi i} \int_{c-i \infty}^{c+i \infty} g(s) t^{-s} d s
$$

for some real $c$ such that the integral converges for all $t>0$.

In general, the Mellin transform is defined only in some region of the complex plane. This region turns out to be a strip, called the fundamental strip (see [35, Definition 1]). If $f(t)=\mathcal{O}_{0}\left(t^{\alpha}\right)$ and $f(t)=\mathcal{O}_{\infty}\left(t^{\beta}\right)$, then $\mathcal{M}[f](s)$ exists at least in the strip $-\alpha<\mathfrak{R}(s)<-\beta$ (cf. [35], Lemma 1). The invertibility of Mellin transform is addressed by the following theorem.

Theorem 2 ([35], Theorem 2 ) Let $f$ be a continuous function. If $c$ is a real number belonging to the fundamental strip of $\mathcal{M}[f]$ and $\mathbb{R} \ni y \mapsto \mathcal{M}[f](c+i y)$ is Lebesgue integrable, then for any $t \in \mathbb{R}^{+}$

$$
f(t)=\mathcal{M}^{-1}[\mathcal{M}[f]](t)=\frac{1}{2 \pi i} \int_{c-i \infty}^{c+i \infty} \mathcal{M}[f](s) t^{-s} d s .
$$

We have established the framework and now we are ready to formulate and prove the main results.

\section{General Results}

The Mellin transform has a direct application to the study of the asymptotic expansions of heat traces. Recall that $P$ is, by General Assumption, a positive densely defined operator with compact inverse. Let us start with the following lemma.

Lemma 1 Let $P$ be such that its zeta-function is well-defined with abscissa of convergence $L$. Then for any $s \in \mathbb{C}$ with $\mathfrak{R}(s)>L$,

$$
\mathcal{M}\left[\operatorname{htr}_{P}\right](s)=\Gamma(s) \zeta_{P}(s)
$$


Proof For $s$ with $\mathfrak{R}(s)>L$ we pick any $\alpha$ such that $\mathfrak{R}(s)>\alpha>L$. From Corollary 3 we know that $\operatorname{htr}_{P}(t)=\mathcal{O}_{0}\left(t^{-\alpha}\right)$ and the integral

$$
\mathcal{M}\left[\operatorname{htr}_{P}\right](s)=\int_{0}^{\infty} \operatorname{htr}_{P}(t) t^{s-1} d t
$$

converges (absolutely) at 0 . It also converges absolutely at $\infty$ for any $s$, since

$$
\operatorname{Tr} \mathrm{e}^{-t P}=\sum_{n=0}^{\infty} M_{n} \mathrm{e}^{-t \lambda_{n}}=\mathrm{e}^{-t \lambda_{0}} \sum_{n=0}^{\infty} M_{n} \mathrm{e}^{-t\left(\lambda_{n}-\lambda_{0}\right)} \leqslant \mathrm{e}^{-t \lambda_{0}} c \text { for } t \geqslant 1,
$$

where $c=\sum_{n=0}^{\infty} M_{n} \mathrm{e}^{-\left(\lambda_{n}-\lambda_{0}\right)}=\mathrm{e}^{\lambda_{0}} \operatorname{Tr} \mathrm{e}^{-P}<\infty$.

Since $M_{n} \mathrm{e}^{-t \lambda_{n}} t^{s-1}>0$ for all $n \in \mathbb{N}, t>0$ and $s$ with $\mathfrak{R}(s)>L$, we can use the Tonelli Theorem to exchange the sum with integral in the following calculation:

$$
\begin{aligned}
\mathcal{M}\left[\operatorname{htr}_{P}\right](s) & =\int_{0}^{\infty} \sum_{n=0}^{\infty} M_{n} \mathrm{e}^{-t \lambda_{n}} t^{s-1} d t=\sum_{n=0}^{\infty} M_{n} \int_{0}^{\infty} \mathrm{e}^{-t \lambda_{n}} t^{s-1} d t \\
& =\sum_{n=0}^{\infty} M_{n} \lambda_{n}^{-s} \int_{0}^{\infty} \mathrm{e}^{-y} y^{s-1} d y=\zeta_{P}(s) \Gamma(s)
\end{aligned}
$$

For further convenience we adopt the notation

$$
\mathcal{Z}(s):=\Gamma(s) \zeta_{P}(s),
$$

for $\mathfrak{R}(s)>L$. If moreover, $\zeta_{P}$ extends to a meromorphic function on some larger region $D \subset \mathbb{C}$ then $\mathcal{Z}$ also has a meromorphic extension to $D$, since $\Gamma$ is meromorphic on $\mathbb{C}$. For a meromorphic function $f$ we also denote by $\mathfrak{P}_{f}(D)$ the set of its poles contained in the region $D \subset \mathbb{C}$.

The inverse of the relation (9) (compare [35, Theorem 5]) produces an expansion of $\operatorname{htr}_{P}$ :

Theorem 3 Let $P$ be an operator satisfying the General Assumption and such that:

1. $\zeta_{P}$ is well-defined with abscissa of convergence $L \geqslant 0$.

2. $\zeta_{P}$ has a meromorphic continuation to the half-plane $\mathfrak{R}(s)>L^{\prime}$ for some real $L^{\prime}<L$.

3. There exist real numbers $c, R$, such that $L^{\prime}<-R<L<c$, and $\mathcal{Z}$ is regular and Lebesgue integrable on lines $\mathfrak{R}(s)=-R$ and $\mathfrak{R}(s)=c$.

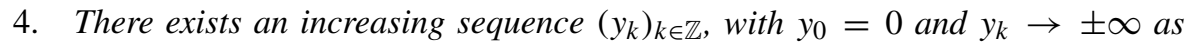
$k \rightarrow \pm \infty$, such that

$$
\sup _{x \in[-R, c]}\left|\mathcal{Z}\left(x+i y_{k}\right)\right| \rightarrow 0
$$

as $k \rightarrow \pm \infty$ and the suprema for all $k \in \mathbb{Z}^{*}$ are finite.

Let $D_{k}$ denote a rectangle $\left\{x+i y:-R \leqslant x \leqslant c, y_{-k} \leqslant y \leqslant y_{k}\right\} \subset \mathbb{C}$ for $k \in \mathbb{N}^{+}$ and $D_{0}=\emptyset$ and let $S_{k}:=\mathfrak{P}_{\mathcal{Z}}\left(D_{k} \backslash D_{k-1}\right)$ (see Fig. 1). 


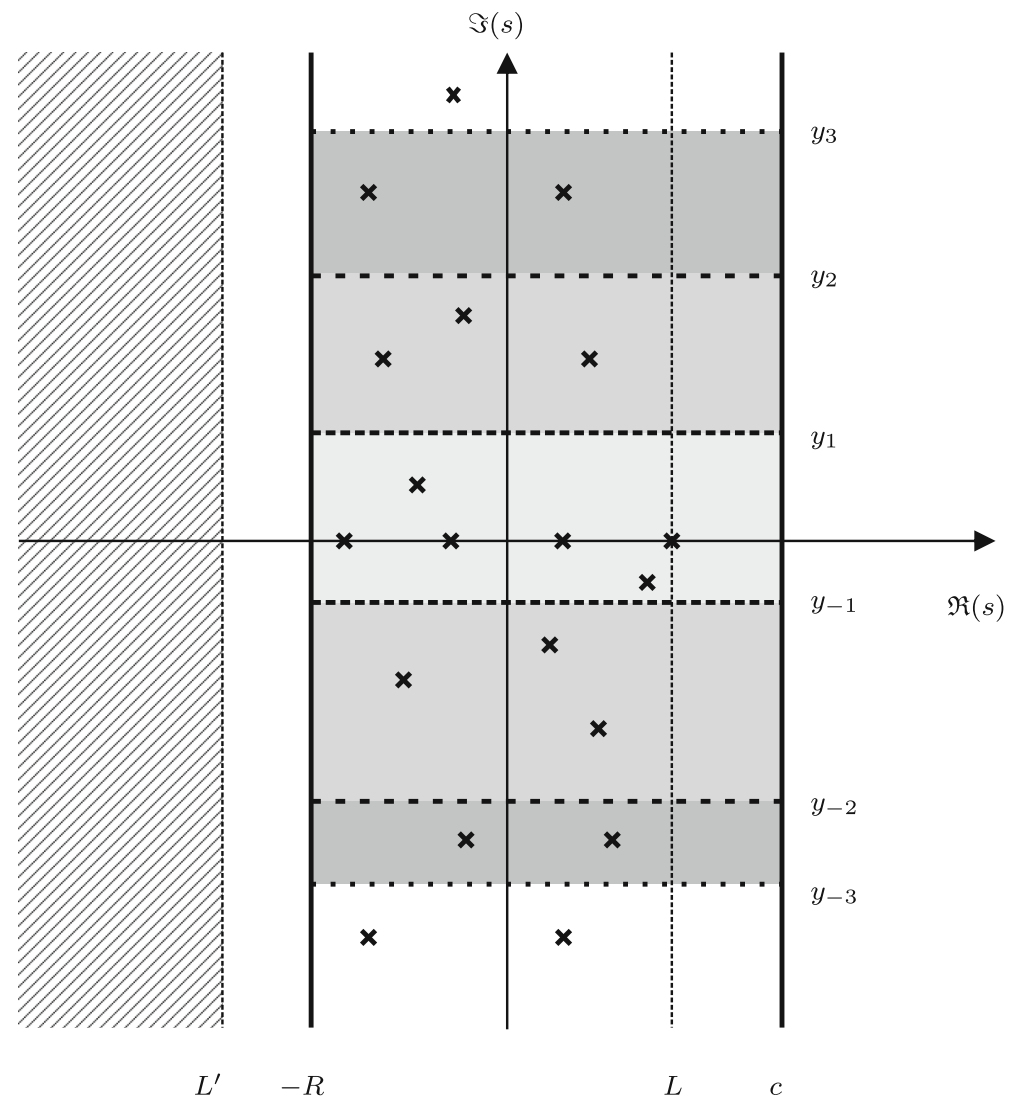

Fig. 1 Illustration for Theorem 3. The crosses stand for poles of the function $\mathcal{Z}$

Then, for $t>0$, we have

$$
\operatorname{htr}_{P}(t)=\sum_{k=1}^{\infty} \sum_{s \in S_{k}} r_{s}(t)+F_{R}(t),
$$

where the, possibly infinite, series over $k$ is convergent with

$$
\begin{aligned}
r_{s}(t) & :=\operatorname{Res}_{s^{\prime}=s}\left(\Gamma\left(s^{\prime}\right) \zeta_{P}\left(s^{\prime}\right) t^{-s^{\prime}}\right), \\
F_{R}(t) & :=\frac{1}{2 \pi i} \int_{-R-i \infty}^{-R+i \infty} \Gamma(s) \zeta_{P}(s) t^{-s} d s .
\end{aligned}
$$

Moreover, $F_{R}(t)=\mathcal{O}_{0}\left(t^{R}\right)$. 
Proof On the strength of Assumptions 3 and 4 the function $\mathcal{Z}$ is regular at the boundary of $D_{k}$. Thus, by the residue theorem,

$$
\frac{1}{2 \pi i} \int_{\partial D_{k}} \mathcal{Z}(s) t^{-s} d s=\sum_{s \in \mathfrak{P}_{\mathcal{Z}}\left(D_{k}\right)} r_{s}(t)=\sum_{m=1}^{k} \sum_{s \in S_{m}} r_{s}(t),
$$

where the contour $\partial D_{k}$ is oriented counter-clockwise. In the sum above only a finite number of residues is taken into account, as the region $D_{k}$ is bounded and the set of poles $\mathfrak{P}_{\mathcal{Z}}\left(D_{k}\right)$ has no accumulation points. Let us decompose the boundary of the rectangle into four sides

$$
\begin{gathered}
I_{-R}(k):=\int_{-R+i y_{-k}}^{-R+i y_{k}} \mathcal{Z}(s) t^{-s} d s, \quad I_{c}(k):=\int_{c+i y_{-k}}^{c+i y_{k}} \mathcal{Z}(s) t^{-s} d s \\
I_{H}^{ \pm}(k):=\int_{-R+i y_{ \pm k}}^{c+i y_{ \pm k}} \mathcal{Z}(s) t^{-s} d s
\end{gathered}
$$

so that

$$
\int_{\partial D_{k}} \mathcal{Z}(s) t^{-s} d s=I_{c}(k)-I_{H}^{+}(k)-I_{-R}(k)+I_{H}^{-}(k) .
$$

We now estimate

$$
\left|I_{H}^{ \pm}(k)\right|=\left|\int_{-R}^{c} \mathcal{Z}\left(x+i y_{ \pm k}\right) t^{-x-i y_{ \pm k}} d x\right| \leqslant \sup _{x \in[-R, c]}\left|\mathcal{Z}\left(x+i y_{ \pm k}\right)\right| \int_{-R}^{c} t^{-x} d x \stackrel{k \rightarrow \infty}{\longrightarrow} 0 .
$$

To analyse the integrals $I_{-R}, I_{c}$ we will use the Assumption 3. Integrability over the line $\mathfrak{R}(s)=c$ allows us to apply Theorem 2, which, together with Lemma 1 , gives us the following limit

$$
\frac{1}{2 \pi i} \lim _{k \rightarrow \infty} I_{c}(k)=\operatorname{htr}_{P}(t) .
$$

On the other hand, integrability of $\mathcal{Z}$ along the line $\mathfrak{R}(s)=-R$ allows us to write

$$
\frac{1}{2 \pi i} \lim _{k \rightarrow \infty} I_{-R}(k)=F_{R}(t) .
$$

Thus, by taking the limit $k \rightarrow \infty$ of (14) we get

$$
\sum_{m=0}^{\infty} \sum_{s \in S_{m}} r_{s}(t)=\lim _{k \rightarrow \infty} \frac{1}{2 \pi i} \int_{\partial D_{k}} \mathcal{Z}(s) t^{-s} d s=\operatorname{htr}_{P}(t)-F_{R}(t) .
$$

To finish the proof we observe that

$$
F_{R}(t) t^{-R}=\frac{1}{2 \pi} \int_{-\infty}^{\infty} \mathcal{Z}(-R+i y) t^{-i y} d y=\frac{1}{2 \pi} \mathcal{F}[y \mapsto \mathcal{Z}(-R+i y)]\left(-\frac{\log t}{2 \pi}\right) \stackrel{t \rightarrow 0}{\longrightarrow} 0,
$$

where $\mathcal{F}$ denotes the Fourier transform, and the limit is a consequence of the Riemann-Lebesgue lemma. 
The series in (13) is just the sum of residues of the function $\mathcal{Z}(s) t^{-s}$ over the poles contained in the strip $-R<\Re(s)<c$ and one could be tempted to write it down as

$$
\sum_{s \in S} r_{s}(t)
$$

where summation goes over $S=\mathfrak{P}_{\mathcal{Z}}(\{s \in \mathbb{C}:-R<\mathfrak{R}(s)<c\})$. Whilst this second form looks simpler, it ignores the information about grouping and arrangement of terms, which may be significant. Indeed, Theorem 3 states that the series over $k$ in formula (13) is convergent, and in general this may only be a conditional convergence. That is why residues have to be grouped into (finite) sums over $S_{k}$, and then summed in the order given by index $k$. The grouping and order are consequences of the choice of the sequence $\left(y_{k}\right)$ and the assumption (12) may fail for a different choice of sequence.

However, if the function $\mathcal{Z}$ has only a finite number of residues in the strip $-R<$ $\mathfrak{R}(s)<c$ or the sum (17) is absolutely convergent then rearrangements of terms are allowed and one can safely write

$$
\operatorname{htr}_{P}(t)=\sum_{s \in S} r_{s}(t)+F_{R}(t)
$$

instead of (13). One clue about the absolute convergence is given by the following result:

Proposition 4 If the hypothesis of Theorem 3 is fulfilled with the assumption (12) altered for a stronger one:

$$
\sum_{k \in \mathbb{Z}^{*}} \sup _{x \in[-R, c]}\left|\mathcal{Z}\left(x+i y_{k}\right)\right|<\infty,
$$

then for any $t>0$

$$
\sum_{k=1}^{\infty}\left|\sum_{s \in S_{k}} r_{s}(t)\right|<\infty
$$

Proof First note that for any $t>0$

$$
\sum_{k=1}^{\infty}\left|\sum_{s \in S_{k}} r_{s}(t)\right|=\frac{1}{2 \pi i} \sum_{k=1}^{\infty}\left|\int_{\partial\left(D_{k} \backslash D_{k-1}\right)} \mathcal{Z}(s) t^{-s} d s\right| .
$$

Now, for any $k \geqslant 1$ we decompose the boundary $\partial\left(D_{k} \backslash D_{k-1}\right)$ as in the proof of Theorem 3 and estimate

$$
\begin{aligned}
\left|\int_{\partial\left(D_{k} \backslash D_{k-1}\right)} \mathcal{Z}(s) t^{-s} d s\right| \leqslant\left|I_{-R}(k)-I_{-R}(k-1)\right|+\left|I_{c}(k)-I_{c}(k-1)\right|+ \\
\quad+\left|I_{H}^{+}(k-1)\right|+\left|I_{H}^{-}(k-1)\right|+\left|I_{H}^{+}(k)\right|+\left|I_{H}^{-}(k)\right|,
\end{aligned}
$$

with the convention $I_{H}^{ \pm}(0):=0$ to comply with $D_{0}=\emptyset$. So the contributions of the horizontal integrals $I_{H}^{ \pm}(k), I_{H}^{ \pm}(k+1)$ add together instead of canceling out as it happened in the proof of Theorem 3. 
Therefore,

$$
\begin{aligned}
\sum_{k=1}^{\infty}\left|\int_{\partial\left(D_{k} \backslash D_{k-1}\right)} \mathcal{Z}(s) t^{-s} d s\right| \leqslant \int_{-R-i \infty}^{-R+i \infty}\left|\mathcal{Z}(s) t^{-s}\right| d s+ \\
+\int_{c-i \infty}^{c+i \infty}\left|\mathcal{Z}(s) t^{-s}\right| d s+2 \sum_{k=1}^{\infty}\left(\left|I_{H}^{+}(k)\right|+\left|I_{H}^{-}(k)\right|\right) .
\end{aligned}
$$

The convergence of the integrals along the vertical lines follows since $\mathcal{Z}$ is Lebesgue integrable (Assumption 3 of Theorem 3).

On the other hand, the sum over horizontal contributions can be estimated as in (15):

$$
\sum_{k=1}^{\infty}\left(\left|I_{H}^{+}(k)\right|+\left|I_{H}^{-}(k)\right|\right) \leqslant \sum_{k \in \mathbb{Z}^{*}} \sup _{x \in[-R, c]}\left|\mathcal{Z}\left(x+i y_{k}\right)\right| \int_{-R}^{c} t^{-x} d x,
$$

which is finite by assumption (19).

In most cases (see Section 4), one can also avoid the grouping of the residues into $S_{k}$ 's, by finding some denser sequence $\left(y_{k}\right)_{k \in \mathbb{Z}}$ such that each $S_{k}$ contains only one pole of $\mathcal{Z}$. Also, if there are poles of $\mathcal{Z}$ lying on a common line $\Im(s)=$ const., one can resort to the more general Theorem 4. However, for a denser sequence the assumptions (12) or (19) of the Theorem 3 may not be fulfilled.

Unfortunately, we were not able to tailor an example, where the analytic structure of $\zeta_{P}$ is such that the grouping or arrangement of the terms are important. It might turn out that the operatorial aspect of heat traces, which leads to a specific subclass of general Dirichlet series, implies that one can always replace formula (13) with (18). However, as the problem is open, we emphasise once again that in general formulae the series in (13) is conditionally convergent only.

It is instructive to write down explicitly an individual term $r_{s}(t)$. If the function $\mathcal{Z}$ has a pole of order $n$ at $s_{0}$, then it has a Laurent expansion $\mathcal{Z}(s)=\sum_{k=-n}^{\infty} b_{k}\left(s_{0}\right)(s-$ $\left.s_{0}\right)^{k}$ in some open punctured disc with the center at $s_{0}$. On the other hand,

$$
t^{-s}=\mathrm{e}^{-s_{0} \log t} \mathrm{e}^{-\left(s-s_{0}\right) \log t}=t^{-s_{0}} \sum_{k=0}^{\infty} \frac{(-\log t)^{k}}{k !}\left(s-s_{0}\right)^{k}, \quad \forall s_{0}, s \in \mathbb{C}, t>0 .
$$

Therefore, the residue $r_{s_{0}}(t)$ being the coefficient in front of $\left(s-s_{0}\right)^{-1}$ in the Laurent expansion of $\mathcal{Z}(s) t^{-s}$ at $s=s_{0}$ reads

$$
r_{s_{0}}(t)=t^{-s_{0}} \sum_{k=0}^{n-1} \frac{b_{-k-1}\left(s_{0}\right)}{k !}(-\log t)^{k} \text {. }
$$

Note that $r_{s_{0}}(t)=\mathcal{O}_{0}\left(t^{-\Re\left(s_{0}\right)}(\log t)^{n-1}\right)$, what also means that $r_{s_{0}}(t)=$ $\mathcal{O}_{0}\left(t^{-\mathfrak{R}\left(s_{0}\right)+\delta}\right)$ for arbitrarily small $\delta>0$.

Remark 1 One could in principle allow the function $\mathcal{Z}$ to have essential singularities as long as they are isolated (see [8, last point on p. 453] for a motivation). For such functions the residue (i.e. the $-1^{\text {st }}$ term of the Laurent expansion) is well-defined. Since $\Gamma$ is a meromorphic function and $s \mapsto t^{-s}$ is an entire one for all $t>0$, the function

$$
s \mapsto \mathcal{Z}(s) t^{-s}
$$


has isolated singularities only. At an essential singularity, the formula (20) yields an infinite series. This series is absolutely convergent for every $t>0$ in the punctured disc of convergence of the Laurent expansion of $\mathcal{Z}$ at $s_{0}$. Indeed, in the interior of this punctured disc the Laurent series of the function (21) is a product of two absolutely convergent series, and as such it is absolutely convergent.

\subsection{Asymptotic Expansions}

Theorem 3 gives us information about the behavior of $\operatorname{htr}_{P}(t)$ at $t=0$ up to the order $t^{R}$. If $\zeta_{P}$ can be meromorphically continued to the whole complex plane and satisfies suitable growth conditions, then Theorem 3 can give us the behavior of $\operatorname{htr}_{P}$ at 0 up to an arbitrary finite order, i.e. an asymptotic expansion. Recall the definition $[22,31,43]$ :

Definition 4 Let $\left(\phi_{n}\right)_{n \in \mathbb{N}}$ be a sequence of functions from $\mathbb{R}^{+}$to $\mathbb{C}$. We call this sequence an asymptotic scale at $t=0$ if for any $n \in \mathbb{N}$ we have $\phi_{n+1}(t)=\mathcal{O}_{0}\left(\phi_{n}(t)\right)$.

For a function $f: \mathbb{R}^{+} \rightarrow \mathbb{C}$ the formal series $\sum_{n=0}^{\infty} \phi_{n}(t)$, with $\left(\phi_{n}\right)_{n \in \mathbb{N}}$ being an asymptotic scale, is called an asymptotic expansion (or asymptotic series) of $f$ at $t=0$ if for any $N \in \mathbb{N}$

$$
f(t)-\sum_{n=0}^{N} \phi_{n}(t)=\mathcal{O}_{0}\left(\phi_{N+1}(t)\right) .
$$

In this case, we write

$$
f(t) \underset{t \downarrow 0}{\sim} \sum_{n=0}^{\infty} \phi_{n}(t) .
$$

Now, by an iterative argument exploiting Theorem 3 we obtain the asymptotic expansion of $\operatorname{htr}_{P}$, which is the main result of the paper:

Theorem 4 Let $P$ be a an operator satisfying the General Assumption and such that:

1. $\zeta_{P}$ is well-defined with abscissa of convergence $L \geqslant 0$.

2. $\zeta_{P}$ has a meromorphic continuation to the whole complex plane.

3. There exists a sequence $\left(R_{n}\right)_{n \in \mathbb{N}}$ of real numbers strictly increasing to infinity, such that $-R_{0}>L,-R_{n}<L$ for $n \geqslant 1$, and for each $n \in \mathbb{N}$ function $\mathcal{Z}(s)=\Gamma(s) \zeta_{P}(s)$ is regular and Lebesgue integrable over the vertical line $\mathfrak{R}(s)=-R_{n}$.

4. For each $n \geqslant 1$ there exists a strictly increasing sequence $\left(y_{k}^{(n)}\right)_{k \in \mathbb{Z}}$ with $y_{0}^{(n)}=$ 0 and $y_{k}^{(n)} \rightarrow \pm \infty$ as $k \rightarrow \pm \infty$ such that

$$
\sup _{x \in\left[-R_{n},-R_{n-1}\right]}\left|\mathcal{Z}\left(x+i y_{k}^{(n)}\right)\right| \rightarrow 0,
$$

as $k \rightarrow \pm \infty$ and the suprema for all $k \in \mathbb{Z}^{*}$ are finite. 
For $n, k \geqslant 1$ let $D_{k}^{n}$ denote a rectangle $\left\{x+i y:-R_{n} \leqslant x \leqslant-R_{n-1}, y_{-k}^{(n)} \leqslant y \leqslant\right.$ $\left.y_{k}^{(n)}\right\} \subset \mathbb{C}$ and $D_{0}^{n}=\emptyset$ for $n \geqslant 1$. Set $S_{k}^{n}=\mathfrak{P}_{\mathcal{Z}}\left(D_{k}^{n} \backslash D_{k-1}^{n}\right)$ (see Figs. 1 and 2 ). Then, for $t>0$

$$
\operatorname{htr}_{P}(t) \underset{t \downarrow 0}{\sim} \sum_{n=1}^{\infty} \sum_{k=1}^{\infty} \sum_{s \in S_{k}^{n}} r_{s}(t),
$$

where

$$
r_{s}(t)=\operatorname{Res}_{s^{\prime}=s}\left(\mathcal{Z}\left(s^{\prime}\right) t^{-s^{\prime}}\right)
$$

The RHS of (22) is to be understood as the asymptotic (formal) series $\sum_{n=1}^{\infty} \phi_{n}(t)$, with $\phi_{n}: \mathbb{R}^{+} \rightarrow \mathbb{C}$ being an asymptotic scale defined by the convergent series

$$
\phi_{n}(t):=\sum_{k=0}^{\infty} \sum_{s \in S_{k}^{n}} r_{s}(t), \quad \text { for } n \geqslant 1 .
$$

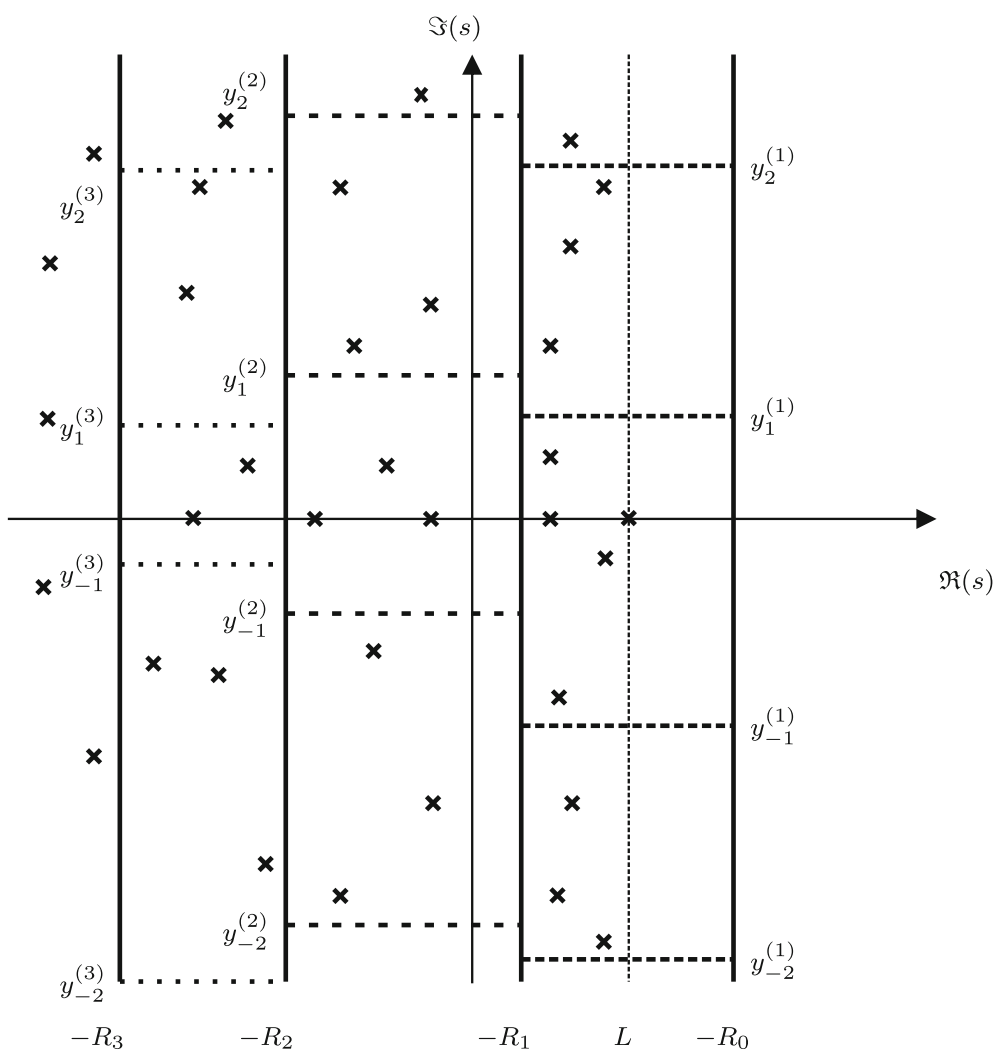

Fig. 2 Illustration for Theorem 4. See also Fig. 1 
Proof Let us first apply Theorem 3 to $\zeta_{P}$ with $c=-R_{0}, R=R_{1}$ and $y_{k}=y_{k}^{(1)}$ for $k \in \mathbb{Z}$. All of the assumptions are readily fulfilled and we obtain

$$
\operatorname{htr}_{P}(t)=\phi_{1}(t)+F_{R_{1}}(t),
$$

where $\phi_{1}$ is given by (23).

Then, we use Theorem 3 again with $c=-R_{1}, R=R_{2}$ and $y_{k}=y_{k}^{(2)}$ for $k \in \mathbb{Z}$. Strictly speaking in this case $c$ is not greater than $L$ as required by Assumption 3 , but as long as $\mathcal{Z}$ is regular and Lebesgue integrable on the lines $\mathfrak{R}(s)=-R_{1}$ and $\mathfrak{R}(s)=-R_{0}$ the same arguments apply. The contour integral, now being $2 \pi i\left(F_{R_{1}}(t)-F_{R_{2}}(t)\right)$, is again equal to the sum of residues, giving

$$
F_{R_{1}}(t)=\phi_{2}(t)+F_{R_{2}}(t),
$$

instead of (24). Repeating this argument for the function $\zeta_{P}$ in each strip $-R_{n+1} \leqslant$ $\mathfrak{R}(s) \leqslant-R_{n}$ we obtain a recurrence relation:

$$
F_{R_{n}}(t)=\phi_{n+1}(t)+F_{R_{n+1}}(t) .
$$

Thus, for any $N \in \mathbb{N}^{+}$we have

$$
\operatorname{htr}_{P}(t)=\sum_{n=1}^{N} \phi_{n}(t)+F_{R_{N}}(t),
$$

where $F_{R_{N}}(t)=\mathcal{O}_{0}\left(t^{R_{N}}\right)$ and by Definition 4 of an asymptotic expansion we conclude that

$$
\operatorname{htr}_{P}(t) \underset{t \downarrow 0}{\sim} \sum_{n=1}^{\infty} \phi_{n}(t) .
$$

The choice of the sequence $\left(R_{n}\right)$ determines the asymptotic scale $\left(\phi_{n}\right)$ of the expansion (22). Thus, in our method the residues are first summed in each vertical strip $\left\{s \in \mathbb{C}:-R_{n+1}<\mathfrak{R}(s)<-R_{n}\right\}$ yielding $\phi_{n}(t)$, and then the contributions from subsequent strips form an asymptotic series.

\subsection{Exact and Almost Exact Expansions}

Having an asymptotic series for $\operatorname{htr}_{P}(t)$, it is natural to ask whether this series converges for some $t$. This can be checked by analysing the limit of $F_{R_{N}}(t)$ as $N \rightarrow \infty$. If this limit exists and $F_{R_{N}} \rightarrow F_{\infty}<\infty$ locally uniformly on the interval $(0, T)$ for some $T>0$, then the series in (22) converges locally uniformly on $(0, T)$ to $\operatorname{htr}_{P}(t)-F_{\infty}(t)$. Note that $F_{\infty}$ is $\mathcal{O}_{0}\left(t^{R}\right)$ for every $R \in \mathbb{R}$. Let us adopt the following definition:

Definition 5 If the series on the RHS of (22) converges (to $\operatorname{htr}_{P}(t)-F_{\infty}(t)$ ) locally uniformly on $(0, T)$ for some $T>0$ then we say that $\mathrm{htr}_{P}$ has an almost exact expansion on $(0, T)$. If moreover $F_{\infty}=0$ identically, then $\operatorname{htr}_{P}$ has an exact expansion on $(0, T)$. 
In Section 4 we will meet various examples of almost exact and exact expansions of heat traces, with $T<\infty$ and $T=\infty$ as well. Let us now give a general sufficient condition for the existence of an exact expansion.

Theorem 5 Let the assumptions of Theorem 4 be fulfilled and let the estimate

$$
\left|\mathcal{Z}\left(-R_{n}+i y\right)\right| \leqslant C_{n} \mathrm{e}^{-\epsilon_{n}|y|}
$$

hold for every $y \in \mathbb{R}$ and $n \in \mathbb{N}$, where $C_{n}, \epsilon_{n}$ are some positive constants for $n \in \mathbb{N}$. Assume moreover that the sequence $\sqrt[R_{n}]{C_{n} / \epsilon_{n}}$ is bounded for $n \in \mathbb{N}$.

Then,

$$
\operatorname{htr}_{P}(t)=\sum_{n=1}^{\infty} \sum_{k=0}^{\infty} \sum_{s \in S_{k}^{n}} r_{s}(t)
$$

for $t \in(0, T)$, where

$$
T:=\left(\limsup _{n \rightarrow \infty} \sqrt[R_{n}]{\frac{C_{n}}{\epsilon_{n}}}\right)^{-1}
$$

and the series over $n$ is locally uniformly convergent on $(0, T)$.

Proof Let us estimate the reminder $F_{R_{n}}(t)$ as follows

$$
\begin{aligned}
\left|F_{R_{n}}(t)\right|=\frac{1}{2 \pi}\left|\int_{-\infty}^{\infty} \mathcal{Z}\left(-R_{n}+i y\right) t^{R_{n}-i y} d y\right| \leqslant & \frac{1}{2 \pi} \int_{-\infty}^{\infty} C_{n} \mathrm{e}^{-\epsilon_{n}|y|} t^{R_{n}} d y \\
& =\frac{C_{n} t_{n}}{2 \pi} 2 \int_{0}^{\infty} \mathrm{e}^{-\epsilon_{n} y} d y=\frac{C_{n} t_{n}}{2 \pi} \frac{2}{\epsilon_{n}}
\end{aligned}
$$

Let $0<T^{\prime}<T$. Then, for any $t \in\left(0, T^{\prime}\right]$

$$
\limsup _{n \rightarrow \infty}\left(t \sqrt[R_{n}]{\frac{C_{n}}{\epsilon_{n}}}\right)=\frac{t}{T} \leqslant \frac{T^{\prime}}{T} .
$$

Hence, for sufficiently large $n$ we have $t \sqrt[R_{n}]{\frac{C_{n}}{\epsilon_{n}}}<a$, where $a \in\left(T^{\prime} / T, 1\right)$ is some constant independent of $t$. Then

$$
\left|F_{R_{n}}(t)\right| \leqslant \frac{C_{n} t^{R_{n}}}{\epsilon_{n} \pi}<\frac{a^{R_{n}}}{\pi} \rightarrow 0,
$$

so $F_{R_{n}}(t)$ tends to 0 uniformly for $t \in\left(0, T^{\prime}\right]$ as $n \rightarrow \infty$. Since $T^{\prime}$ can be any number in $(0, T)$, the theorem is proven.

Note that again the order of summation in (27) is important and the convergence may be only conditional. As in the case of the vertical sum over $k$ (see (17)), the convergence properties depend on the choice of the $\left(R_{n}\right)$ sequence. And as in Proposition 4 we can refine the assumptions of Theorem 5 to obtain an absolute convergence of the series over $n$ in formula (27).

Let us first adopt the following definition: 
Definition 6 Let the operator $P$ be such that

$$
\operatorname{htr}_{P}(t)=\sum_{n=1}^{\infty} \phi_{n}(t), \quad \text { for } t \in(0, T),
$$

with some $0<T \leqslant+\infty$, where $\left(\phi_{n}\right)_{n \in \mathbb{N}}$ is the asymptotic scale given by (23). We say that the heat trace associated with $P$ has an absolutely exact expansion on $(0, \widetilde{T})$ if the series

$$
\sum_{n=1}^{\infty}\left|\phi_{n}(t)\right|
$$

is locally uniformly convergent on $(0, \widetilde{T})$ with some $0<\widetilde{T} \leqslant T$.

Note, that since the RHS of (29) is not in general a Taylor series, its domain of convergence does not necessarily coincide with that of absolute convergence, hence $\widetilde{T} \leqslant T$. In particular if $0=\widetilde{T}<T$, then the expansion of the heat trace associated with $P$ will be exact on $(0, T)$, but nowhere absolutely exact.

By using a similar reasoning as the one used in the proof of Proposition 4, we can refine the assumptions of Theorem 5 to obtain an absolutely exact heat trace expansion.

Proposition 5 Let the assumptions of Theorem 5 be fulfilled and moreover let $\log n=\mathcal{O}_{\infty}\left(R_{n}\right)$ (i.e. $R_{n}$ grow faster than $\log n$ ). Then the expansion is absolutely exact on the whole domain $(0, T)$.

Proof As announced, we proceed similarly to the proof of Proposition 4, but now we shall add together the contributions of subsequent vertical integrals. From (25) we have

$$
\left|\phi_{n}(t)\right|=\left|F_{R_{n}}(t)-F_{R_{n-1}}(t)\right| \leqslant\left|F_{R_{n}}(t)\right|+\left|F_{R_{n-1}}(t)\right| .
$$

Now, (28) implies

$$
\sum_{n=1}^{\infty}\left|\phi_{n}(t)\right| \leqslant 2 \sum_{n=1}^{\infty}\left|F_{R_{n}}(t)\right| \leqslant 2 \sum_{n=1}^{\infty} \frac{C_{n} t^{R_{n}}}{\epsilon_{n} \pi}<\frac{2}{\pi} \sum_{n=1}^{\infty} a^{R_{n}} .
$$

with $a \in\left(T^{\prime} / T, 1\right)$ for any $T^{\prime} \in(0, T)$. Therefore, it suffices to show that the last series is convergent for any $a<1$. Taking $x=-\log a$ we see that it is again a general Dirichlet series (5) with $a_{n}=1, b_{n}=R_{n}$ for $n \in \mathbb{N}$. By Theorem 1 we conclude that its abscissa of convergence is

$$
\limsup _{n \rightarrow \infty} \frac{\log n}{R_{n}}
$$

which equals 0 by hypothesis. Thus, the series is convergent for $x>0$, i.e $a<1$.

The characterisation of an absolutely exact expansion through Proposition 5 is particularly useful. If we have an exact expansion of a heat $\operatorname{trace~} \operatorname{htr}_{P}$ on $(0, T)$, it is sufficient to check whether the sequence $\left(R_{n}\right)$ of our choice grows faster than $\log n$. 
In fact, all of the examples of exact expansions presented in Section 4 are actually absolutely exact in the same domain.

Let us also note, that if we have an exact expansion of the heat trace for an open interval $(0, T)$, then $\operatorname{htr}_{P}$ actually provides an analytic continuation of the RHS of (27) to the whole half line $\mathbb{R}^{+}$.

Let us now turn to the case of an almost exact expansion of heat traces. The situation is somewhat different than that of an exact expansion, as one would need precise formulae rather than estimates to guarantee that the limit of $F_{R_{N}}$ as $N \rightarrow \infty$ is finite, but non-zero. It may be seen as a kind of "critical" case, in the sense that a slight perturbation of the zeta-function renders the expansion divergent (see Section 4.1.4). One of these specific cases is captured by the following proposition.

Proposition 6 If the operator $P$ fulfilling the assumptions of Theorem 4 is such that $\mathfrak{P}_{\mathcal{Z}}(\mathbb{C})$ is a finite set, then the expansion

$$
\operatorname{htr}_{P}(t)=\sum_{s \in \mathfrak{P}_{\mathcal{Z}}(\mathbb{C})} r_{s}(t)+F_{\infty}(t)
$$

is almost exact for all $t>0$, but not exact.

Proof Let us note that $\mathfrak{P}_{\mathcal{Z}}(\mathbb{C})$ being finite requires $\mathfrak{P}_{\zeta_{P}}(\mathbb{C})$ to be finite, but also that the zeros of $\zeta_{P}$ cancel all but a finite number of poles of $\Gamma$, i.e. $\zeta_{P}(-n)=0$ for all but a finite number of $n \in \mathbb{N}$. In this case, Theorem 4 yields an asymptotic expansion, which has only a finite number of terms, hence it converges for all $t>0$. As $t$ tends to infinity we have form (10) that $\operatorname{htr}_{P}(t)=\mathcal{O}_{t \rightarrow \infty}\left(\mathrm{e}^{-t \lambda_{0}}\right)$. But this is not compatible with the behaviour $t^{-s}(\log t)^{k}$ (compare (20)) of the summands of the first term on the RHS of (30). Hence, $F_{\infty}(t)$ cannot be 0 and the expansion (30) is almost exact, but not exact.

\subsection{Truncated Zeta-function}

For further purposes it is convenient to define also the following spectral function.

Definition 7 For any $N \in \mathbb{N}^{+}$, a truncated zeta-function associated with the operator $P$ is a complex function

$$
\mathbb{C} \supset \operatorname{Dom}\left(\zeta_{P}\right) \ni s \mapsto \hat{\zeta}_{P}^{N}(s)=\sum_{n=N}^{\infty} M_{n} \lambda_{n}^{-s} .
$$

The two zeta-functions are related by

$$
\zeta_{P}(s)=\sum_{n=0}^{N-1} M_{n} \lambda_{n}^{-s}+\hat{\zeta}_{P}^{N}(s) .
$$

The first term of the RHS of the above formula is an entire function of $s$, hence the analytic properties of $\zeta_{P}$ and $\hat{\zeta}_{P}^{N}$ are identical. 
We can actually slightly relax the growth rate Assumptions 3 and 4 of Theorem 4 by considering the truncated zeta-function (31) at the place of $\zeta_{P}$.

Proposition 7 If the assumptions of Theorem 3 hold for $\hat{\zeta}_{P}^{N}$ and $\hat{\mathcal{Z}}: s \mapsto \Gamma(s) \hat{\zeta}_{P}^{N}(s)$ with some finite $N \in \mathbb{N}^{+}$, then for $t>0$ we have

$$
\operatorname{htr}_{P}(t)=\sum_{n=0}^{N-1} M_{n} \mathrm{e}^{-t \lambda_{n}}+\sum_{n=0}^{\infty} \sum_{s \in S_{n}} \hat{r}_{s}(t)+\hat{F}_{R}(t),
$$

where

$$
\begin{aligned}
\hat{r}_{s}(t) & :=\operatorname{Res}_{s^{\prime}=s}\left(\hat{\mathcal{Z}}\left(s^{\prime}\right) t^{-s^{\prime}}\right), \\
\hat{F}_{R}(t) & :=\frac{1}{2 \pi i} \int_{-R-i \infty}^{-R+i \infty} \hat{\mathcal{Z}}(s) t^{-s} d s=\mathcal{O}_{0}\left(t^{R}\right) .
\end{aligned}
$$

Proof The proof of Theorem 3 will work equally well for this case with the only difference at line (16) which will now read

$$
\frac{1}{2 \pi i} \lim _{n \rightarrow \infty} \hat{I}_{c}(n)=\mathcal{M}^{-1}[\hat{\mathcal{Z}}](t) .
$$

Applying the inverse Mellin transform to relation (32) multiplied by $\Gamma(s)$ we get (using Lemma 1)

$$
\operatorname{htr}_{P}(t)=\sum_{n=0}^{N-1} M_{n} \mathcal{M}^{-1}\left[\lambda_{n}^{-s} \Gamma(s)\right]+\mathcal{M}^{-1}[\hat{\mathcal{Z}}](t) .
$$

Note that for any $n \in \mathbb{N}$ the function $\lambda_{n}^{-s} \Gamma(s)$ is integrable over any vertical line $\mathfrak{R}(s)=c \notin \mathbb{N}$ (as $\Gamma$ decays exponentially on verticals, compare (52)) and that it equals to $\mathcal{M}\left[\exp \left(-t \lambda_{n}\right)\right](s)$ (cf. (11)). The claim then follows from Theorem 2.

As $\zeta_{P}$ and $\hat{\zeta}_{P}^{N}$ differ by an entire function, the assumptions $(i)$ and (ii) of Theorem 3 are fulfilled by $\hat{\zeta}_{P}^{N}$ if and only if they are fulfilled by $\zeta_{P}$. In view of Proposition 7 it is convenient to introduce the notion of a truncated heat trace:

$$
\operatorname{htr}_{P}^{N}(t):=\operatorname{htr}_{P}(t)-\sum_{n=0}^{N-1} M_{n} \mathrm{e}^{-t \lambda_{n}} .
$$

Then all of the considerations about the truncated zeta-function can be expressed in one simple remark:

Remark 2 All of the results concerning the asymptotic and exact expansions of heat traces hold with $\zeta_{P}$ altered for the truncated zeta-function $\hat{\zeta}_{P}^{N}(31)$. One proceeds as in Proposition 7 and substitutes $\operatorname{htr}_{P}^{N}$ for $\operatorname{htr}_{P}$ and $\hat{\mathcal{Z}}$ for $\mathcal{Z}$ in all of the assertions.

The truncation procedure proves very useful when some of the assumptions on the zeta-function are not met, because of a finite number of terms in its definition (or 
because of a finite number of eigenvalues of $P$ ). We will encounter one such situation when discussing operators with polynomial spectrum in the next section. Zetafunctions of such operators do not generally satisfy the assumption of integrability on vertical lines (Assumption 3 of Theorems 3 and 4), however they can always be truncated to get an asymptotic expansion of the truncated heat trace (see Theorem 7).

Another situation when truncation is beneficial is when the operator $P$ under consideration is not positive or invertible, but there exists such a real constant $c$, that $P+c$ satisfies the General Assumption ( $P$ still has to be self-adjoint and have compact resolvent). Such a $P$ has a finite number of non-positive eigenvalues and we can truncate them. The heat $\operatorname{trace~htr~}_{P}$ still may be defined, although the definition of zeta-function $\zeta_{P}$ is ambiguous at best. However, $\hat{\zeta}_{P}^{N}$ makes sense for $N$ such that for every $n \geqslant N$ one has $\lambda_{n}>0$, if only its abscissa of convergence is finite. Then one can obtain the asymptotic expansion of $\operatorname{htr}_{P}$ from Proposition 7. An important special case is the following:

Remark 3 Let $P$ be a positive densely defined operator with compact resolvent and non-trivial kernel, acting on a separable infinite-dimensional Hilbert space. Let $\left(\lambda_{n}\right)_{n=0}^{\infty}$ denote the strictly increasing sequence of its eigenvalues, that tends to infinity. Then $\lambda_{0}=0$ with $M_{0}=\operatorname{dim} \operatorname{ker} P<\infty$ and every other eigenvalue is positive. If $\operatorname{htr}_{P}$ is well-defined then from (33) one obtains

$$
\operatorname{htr}_{P}(t)=\operatorname{dim} \operatorname{ker} P+\operatorname{htr}_{P}^{1}(t)
$$

and one can apply the Mellin transform techniques to obtain the asymptotic expansion of $\hat{h t r}_{P}^{1}(t)$ if only $\hat{\zeta}_{P}^{1}$ is well-defined and possesses the required properties.

\section{Examples}

\subsection{Operators of Polynomial Spectrum}

In this section we investigate heat traces associated with operators, the eigenvalues and multiplicities of which are given by polynomials. For brevity we shall call such operators - the operators of polynomial spectrum. They appear naturally in the context of Dirac and Laplace operators on spheres [6, 69] and their isospectral deformations [17, 18, 24-26, 62].

Moreover, the results presented in this section apply almost directly when the spectra of the relevant operators can be written as

$$
\sigma(P)=\bigcup_{k=1}^{K}\left\{\lambda_{n}^{k}: n \in \mathbb{N}\right\},
$$

for some finite $K \in \mathbb{N}^{+}$, and for each $k \in\{1, \ldots, K\}$ the eigenvalues $\lambda_{n}^{k}$ and their respective multiplicities $M_{n}^{k}$ are given by polynomials in $n$. Since the (inverse) Mellin transform is linear, one can apply the general theory to each sequence $\left(\lambda_{n}^{k}\right)_{n \in \mathbb{N}}$ separately. One can find examples of such spectra in the framework of Dirac operators on some homogeneous spaces [3, 57, 68], like the 
Poincaré sphere or lens spaces. Indeed, [57, (6.3), (6.11)] give the decomposition of Dirac spectrum into four ${ }^{1}(K=4)$ polynomial sequences for quaternionic space $S U(2) / Q 8$. The same paper describes the spectrum of Poincare sphere with $K=120$ [57, Prop. 7.2] and of four-dimensional lens space $S U(2) / \mathbb{Z}_{4}$ with $K=4$ [57, Lemma 11.20].

By combining the results of [59] with general theorems presented in the preceding section we are able to prove the existence of an asymptotic expansion of the heat traces associated with operators of polynomial spectra (see Theorem 7). We also derive sufficient conditions for the convergence of the expansion.

\subsubsection{Asymptotic Expansions}

Let us start with the following theorem summarising the behaviour of zeta-functions associated with operators in the considered class (compare [59, Theorems A and B]).

Theorem 6 Let $P$ be an operator satisfying the General Assumption with eigenvalues $\lambda_{n}=A(n)$ and multiplicities $M_{n}=B(n)$ for $n \in \mathbb{N}$, where $A$ and $B$ are polynomials.

Then:

1. Roots of $A$ are not in $\mathbb{N}$;

2. $\zeta_{P}$ is well-defined, with the abcissa of convergence $L=(1+\operatorname{deg} B) / \operatorname{deg} A$;

3. $\zeta_{P}$ admits a meromorphic extension to the whole complex plane;

4. $\mathfrak{P}_{\zeta_{P}}(\mathbb{C}) \subset \frac{1}{\operatorname{deg} A}(1+\operatorname{deg} B-\mathbb{N}) \backslash(-\mathbb{N})$ and all of the poles are of first order;

5. For any $\epsilon>0$ there exists $N>0$ such that the truncated zeta-function (see (31)), $\hat{\zeta}_{P}^{N}$, obeys the following growth rate along the vertical lines

$$
\left|\hat{\zeta}_{P}^{N}(x+i y)\right|=\mathcal{O}_{\infty}\left(\mathrm{e}^{\epsilon|y|}\right)
$$

for any $x \in \mathbb{R}$.

Proof The point 1 is a direct consequence of the fact, that $P$ is invertible, and hence all its eigenvalues are non-zero. Now, the zeta-function associated with the operator $P$ for $\mathfrak{R}(s)>L$ can be written as

$$
\zeta_{P}(s)=\sum_{n=0}^{\infty} \frac{B(n)}{A(n)^{s}},
$$

since the roots of $A$ are not in $\mathbb{N}$. The corresponding spectral growth function reads

$$
N\left(\lambda_{n}\right)=\sum_{k=0}^{n} B(k)=: \widetilde{B}(n),
$$

\footnotetext{
${ }^{1}$ To be precise, eigenvalues and multiplicities are in [57] indexed by $n \in \mathbb{Z}$, and hence they need only $K / 2$ polynomials. In our convention with $n \in \mathbb{N}$ we need separate polynomials for positive and negative part of spectrum, what doubles the number of polynomials given in [57].
} 
and it is a classical result (see e.g. the Faulhaber's formula) that $\widetilde{B}$ is a polynomial and $\operatorname{deg} \widetilde{B}=\operatorname{deg} B+1$. Hence, by Theorem 1 the abscissa of convergence of $\zeta_{P}$ equals (cf. (8))

$$
L=\limsup _{n \rightarrow \infty} \frac{\log N\left(\lambda_{n}\right)}{\log \lambda_{n}}=\limsup _{n \rightarrow \infty} \frac{\log \widetilde{B}(n)}{\log A(n)}=\frac{\operatorname{deg} B+1}{\operatorname{deg} A} .
$$

Point 3 is the content of Theorem B in [59] (see also Remark 1 therein).

To prove points 4 and 5 we introduce for $r \in \mathbb{N}^{+}$the following multi-variable series (see [59, Section 1, Formula (2)])

$$
\zeta_{r}\left(s_{1}, \ldots, s_{r} ; \alpha_{1}, \ldots, \alpha_{r}\right):=\sum_{n=0}^{\infty}\left(n+\alpha_{1}\right)^{-s_{1}}\left(n+\alpha_{2}\right)^{-s_{2}} \cdots\left(n+\alpha_{r}\right)^{-s_{r}},
$$

for $s_{1}, \ldots, s_{r} \in \mathbb{C}$ with $\mathfrak{R}\left(s_{1}+\ldots+s_{r}\right)>1$ and $\alpha_{1}, \ldots, \alpha_{r} \in \mathbb{C} \backslash(-\mathbb{N})$. The branch of logarithm in $\left(n+\alpha_{j}\right)^{-s_{j}}=\exp \left(-s_{j} \log \left(n+\alpha_{j}\right)\right)$ is chosen to be $-\pi<$ $\arg \left(n+\alpha_{j}\right) \leqslant \pi$.

If we take $r=\operatorname{deg} A$ and $\alpha_{i}$ for $i=1, \ldots, \operatorname{deg} A$ to be the roots of $A$, then we can write the polynomials $A$ and $B$ as

$$
A(n)=a \prod_{i=1}^{\operatorname{deg} A}\left(n+\alpha_{i}\right), \quad B(n)=\sum_{j=0}^{\operatorname{deg} B} \widetilde{b}_{j}\left(n+\alpha_{1}\right)^{j} .
$$

Plugging this representation into (35) and using the definition (37) we get (compare [59, Section 1, Formula (3)])

$$
\zeta_{P}(s)=a^{-s} \sum_{j=0}^{\operatorname{deg} B} \widetilde{b}_{j} \zeta_{\operatorname{deg} A}\left(s-j, s, \ldots, s ; \alpha_{1}, \ldots, \alpha_{\operatorname{deg} A}\right) .
$$

In [59] the analytic properties of $\zeta_{r}$ functions are studied, what allows to draw conclusions about the analytic properties of $\zeta_{P}$ on the strength of formula (38). Formulae (10) and (12) in [59, Section 1] provide an explicit meromorphic continuation of $\zeta_{r}$ to $\mathbb{C}^{r}$. The proof is based on the induction on $r$ with the Riemann zeta-function as the starting point $r=1$.

The claim $\mathfrak{P}_{\zeta_{P}}(\mathbb{C}) \subset \frac{1}{\operatorname{deg} A}(1+\operatorname{deg} B-\mathbb{N})$ then follows from [59, Theorem A]. On the other hand, [59, Theorem $\mathrm{C}]$ implies that $-\mathbb{N} \nsubseteq \mathfrak{P}_{\zeta_{P}}(\mathbb{C})$. The fact that the poles $\zeta_{P}$ are at most of first order is a consequence of [59, Lemma 5] and the formula [59, Section 1, Formula (12)] (see also [59, Section 1.2 and p. 242]).

Let us now pass on to the last point of the claim - the estimate of $\hat{\zeta}_{P}^{N}$ on vertical lines. We first quote the result [59, Proposition 1 (iii)] translated to our notation:

$$
\left|\zeta_{r}\left(x_{1}+i y_{1}, \ldots, x_{r}+i y_{r} ; \alpha_{1}, \ldots, \alpha_{r-1}, 0\right)\right|=\mathcal{O}_{\infty}\left(C\left(y_{1}, \ldots, y_{r}\right) \cdot \mathrm{e}^{\rho_{1}\left|y_{1}\right|+\ldots+\rho_{r-1}\left|y_{r-1}\right|}\right),
$$

with $\rho_{i}=\left|\arg \alpha_{i}\right|$ and $C-$ a polynomial. Now, we repeat the proof of Proposition 1 (iii) $[59$, p. 240] with the formula (12) instead of (10) therein. The reasoning goes 
along the same lines, [59, Lemma 1] still applies with $y=\Im(z), A=-\frac{\pi}{2}, \alpha=$ $y_{r}, B=\rho_{r}-\frac{\pi}{2}, \beta=0$ and we conclude that

$\left|\zeta_{r}\left(x_{1}+i y_{1}, \ldots, x_{r}+i y_{r} ; \alpha_{1}, \ldots, \alpha_{r-1}, \alpha_{r}\right)\right|=\mathcal{O}_{\infty}\left(C\left(y_{1}, \ldots, y_{r}\right) \cdot \mathrm{e}^{\rho_{1}\left|y_{1}\right|+\ldots+\rho_{r-1}\left|y_{r-1}\right|+\rho_{r}\left|y_{r}\right|}\right)$.

Hence, by (38) we obtain

$\left|\zeta_{P}(x+i y)\right|=\mathcal{O}_{\infty}\left(C(y)^{\operatorname{deg} A} \cdot \mathrm{e}^{|y| \sum_{i=1}^{\operatorname{deg} A}\left|\arg \alpha_{i}\right|}\right)=\mathcal{O}_{\infty}\left(\mathrm{e}^{|y|\left(\sum_{i=1}^{\operatorname{deg} A}\left|\arg \alpha_{i}\right|+\delta\right)}\right)$,

for any $\delta>0$.

Here comes the advantage of using the truncated zeta-function $\hat{\zeta}_{P}^{N}$, since by starting the zeta-series at $n=N$ instead of $n=0$ we effectively shift $\alpha_{i} \rightarrow \alpha_{i}+N$ (compare [59, Remark 1]). This means that by taking $N$ large enough one can make $\sum_{i=1}^{\operatorname{deg} A}\left|\arg \alpha_{i}\right|$ arbitrarily small and assertion 5 is proven.

Typically (i.e. for generic polynomials $A$ and $B$ ) the zeta-functions $\zeta_{P}$ will have an infinite number of poles on the negative part of the real axis. This is a consequence of the [59, formulae (10) and (12)]. Let us illustrate this property with the following simple example.

Example 1 Let $P$ be an operator with eigenvalues $\lambda_{n}=A(n)=n(n+\alpha)$ for $n \in \mathbb{N}^{+}$ with some $\alpha \in \mathbb{R}^{+}$and no degeneracies (i.e. $B(n)=1$ ), then $\mathfrak{P}_{\zeta_{P}}(\mathbb{C})=\frac{1}{2}-\mathbb{N}$.

Indeed, from the formulae (38) and [59, (10)] we have for $\mathfrak{R}(s)>-M / 2$ with any $M \in \mathbb{N}$ :

$$
\zeta_{P}(s)=\sum_{j=0}^{M}\left(\begin{array}{c}
-s \\
j
\end{array}\right) \zeta(2 s+j) \alpha^{j}+h_{M}(s)
$$

where $h_{M}$ is a remainder term holomorphic for $\mathfrak{R}(s)>-M / 2$. So for any $n \in \mathbb{N}$ we have

$$
\operatorname{Res}_{s=\frac{1}{2}-n} \zeta_{P}(s)=\operatorname{Res}_{s=\frac{1}{2}-n}\left(\sum_{j=0}^{2 n}\left(\begin{array}{c}
-s \\
j
\end{array}\right) \zeta(2 s+j) \alpha^{j}+h_{2 n}(s)\right)=\left(\begin{array}{c}
n-\frac{1}{2} \\
2 n
\end{array}\right) \alpha^{2 n} \neq 0 .
$$

There exist however special operators $P$ with fine-tuned polynomials $A$ and $B$ for which the zeta-function $\zeta_{P}$ will only have a finite number of poles. It happens for instance in the following case:

Proposition 8 If all of the roots of the polynomial $A$ are equal then the $\operatorname{set} \mathfrak{P}_{\zeta_{P}}(\mathbb{C})$ is finite. 
Proof Since $A(n)=a(n+\alpha)^{\operatorname{deg} A}$, we have for $\Re(s)>(1+\operatorname{deg} B) / \operatorname{deg} A$

$$
\zeta_{P}(s)=\sum_{n=0}^{\infty} \sum_{j=0}^{\operatorname{deg} B} \tilde{b}_{j} a^{-s}(n+\alpha)^{-(\operatorname{deg} A) s+j}=a^{-s} \sum_{j=0}^{\operatorname{deg} B} \tilde{b}_{j} \zeta_{H}((\operatorname{deg} A) s-j, \alpha),
$$

where $\zeta_{H}$ is the Hurwitz zeta-function. Since $\zeta_{H}$ is meromorphic on $\mathbb{C}$ with a single simple pole at 1 and the sum over $j$ for $\zeta_{P}$ is finite, the assertion follows.

The equality of all roots of $A$ is a sufficient condition for $\mathfrak{P}_{\zeta_{P}}(\mathbb{C})$ to be finite, but not a necessary one. Consider for instance the operator $P$ with eigenvalues $\lambda_{n}=$ $A(n)=n^{3}+1$ and degeneracies $B(n)=n^{2}$. Then, by using

$$
(1+x)^{-s}=\sum_{k=0}^{\infty}\left(\begin{array}{c}
-s \\
k
\end{array}\right) x^{k}, \text { for }|x|<1,
$$

we obtain

$$
\zeta_{P}(s)=\sum_{n=0}^{\infty} \frac{n^{2}}{\left(n^{3}+1\right)^{s}}=\sum_{n=0}^{\infty} n^{-3 s+2}\left(1+n^{-3}\right)^{-s}=\sum_{j=0}^{\infty}\left(\begin{array}{c}
-s \\
j
\end{array}\right) \zeta(3 s+3 j-2),
$$

which gives a meromorphic extension of $\zeta_{P}$ to the whole complex plane. On the other hand, the Theorem 6 assertion 4 implies that $\mathfrak{P}_{\zeta_{P}}(\mathbb{C}) \subset \frac{1}{3}(3-\mathbb{N}) \backslash(-\mathbb{N})$. Moreover, the poles of $\zeta_{P}$ come only from the poles of $s \mapsto \zeta(3 s+3 j-2)$. But at $s=\frac{k}{3}-n, \zeta(k-3 n+3 j-2)$ is finite for all $n \in \mathbb{N}, j \in \mathbb{N}$ and $k \in\{1,2\}$ since the only pole of the Riemann zeta-function is at 1 . Hence, $\zeta_{P}$ has only one simple pole $\mathfrak{P}_{\zeta_{P}}(\mathbb{C})=\{1\}$.

It is interesting to compare this result with [40, Lemma 1.10.1] and [39, p. 2]. The former tells us that zeta-functions associated with classical positive elliptic differential operators on (finite-dimensional) compact manifolds have a finite number of poles only. On the other hand, the latter says that positive elliptic classical pseudodifferential operators generically do have an infinite number of simple poles, including at $-\mathbb{N}$.

Clearly, elliptic pseudodifferential operators on compact manifolds need not be of polynomial spectrum - for instance, the scalar Laplacian on $\mathbb{T}^{2}$ [32]. On the other hand, one might ask whether any operator of polynomial spectrum can be realised as a classical elliptic pseudodifferential operator on some compact manifold. We consider it as an interesting open problem, the solution to which may shed more light on the geometrical meaning of the operators in this class.

Finally, let us turn to the heat traces of operators with eigenvalues and multiplicites given by polynomials. The following Theorem establishes the existence of an asymptotic expansion of heat trace for any operator in this class.

Theorem 7 Let $P$ be an operator with eigenvalues $\lambda_{n}=A(n)$ and multiplicities $M_{n}=B(n)$, where $A$ and $B$ are polynomials of degree $\operatorname{deg} A$ and $\operatorname{deg} B$ respectively. 
Then, there exists $N \in \mathbb{N}$ such that

$$
\operatorname{htr}_{P}^{N}(t)=\operatorname{htr}_{P}(t)-\sum_{n=0}^{N-1} B(n) \mathrm{e}^{-A(n) t} \underset{t \downarrow 0}{\sim} \sum_{k=0}^{\infty} \operatorname{Res}_{s=\ell-k /(\operatorname{deg} A)}\left(\Gamma(s) \hat{\zeta}_{P}^{N}(s)\right) t^{\ell-k /(\operatorname{deg} A)},
$$

where $\ell=(1+\operatorname{deg} B) /(\operatorname{deg} A)$.

Proof The claim follows from a direct application of Theorem 4 together with Remark 2. The assumptions are met by Theorem 6 .

Remark 4 Let us remark that one could actually extend Theorem 6 to operators, the eigenvalues and multiplicites of which can be written as

$$
\lambda_{n}=a n^{\gamma_{0}} \prod_{i=1}^{\operatorname{deg} A}\left(n^{\gamma_{i}}+\alpha_{i}\right), \quad M_{n}=\sum_{j=0}^{\operatorname{deg} B} b_{j} n^{\beta_{j}},
$$

with $\gamma_{i}>0$ for $i=1, \ldots, \operatorname{deg} A, \sum_{i=0}^{\operatorname{deg} A} \gamma_{i}>0$ and $b_{j}>0$ for at least one $j \in\{0, \ldots, \operatorname{deg} B\}$. Then, instead of (37), one would need to seek a meromorphic continuation to $\mathbb{C}^{r}$ of the functions

$\widetilde{\zeta}_{r}\left(s_{1}, \ldots, s_{r} ; \gamma_{0}, \ldots, \gamma_{r} ; \alpha_{1}, \ldots, \alpha_{r}\right):=\sum_{n=0}^{\infty} n^{-s_{0} \gamma_{0}}\left(n^{\gamma_{1}}+\alpha_{1}\right)^{-s_{1}} \cdots\left(n^{\gamma_{r}}+\alpha_{r}\right)^{-s_{r}}$.

The latter could again be accomplished with the help of the Mellin-Barnes formula as in [59]. It is plausible that $\zeta_{P}$ functions obtained in this way will only have first order isolated poles and some bound similar to (39) can be established. On the other hand, the regularity of $\zeta_{P}$ at $-\mathbb{N}$ is not to be expected in general.

We have chosen to formulate Theorem 6 for operators of polynomial spectrum, as considering more general ones described above would add to the complexity without being strongly motivated. In fact, we were not able to find any reasonable geometric example where the operator falls into this larger class, but not the one of operators of polynomial spectrum.

Generically, the expansion (40) will only be an asymptotic one. There exist, however, operators for which the formula (40) will be exact or almost exact on $(0, T)$ for some $0<T \leqslant+\infty$.

\subsubsection{Exact Expansions}

In this subsection we consider a class of operators, which have an exact expansion of the associated heat traces. In particular, it will serve as an illustration for Theorem 5 .

Proposition 9 Let $P$ be an operator of polynomial spectrum with eigenvalues $\lambda_{n}=$ $A(n)$ and multiplicities $M_{n}=B(n)$. If $\operatorname{deg} A=1$, i.e. if $A(n)=a(n+\alpha)$ for some $a>0, \alpha \geqslant 0$, then the asymptotic expansion (40) is absolutely exact on $(0,2 \pi / a)$. 
Proof To start, let us consider an operator $P_{0}$ with $a=1, \alpha=1$ and $B(n)=1$. We can calculate directly:

$$
\zeta_{P}(s)=\sum_{n=1}^{\infty} n^{-s}=\zeta(s), \text { for } \mathfrak{R}(s)>1 .
$$

The Riemann zeta-function $\zeta$ extends meromorphically to $\mathbb{C}$ with a single simple pole at 1 , whereas $\Gamma$ has simple poles at non-positive integer numbers. The values of the residues read

$$
\operatorname{Res}_{s=1} \zeta(s)=1, \quad \operatorname{Res}_{s=-n} \Gamma(s)=\frac{(-1)^{n}}{n !} \forall n \in \mathbb{N} .
$$

Moreover, the values of $\zeta$ at non-positive integers are given by

$$
\zeta(-n)=-\frac{B_{n+1}}{n+1}, \quad \forall n \in \mathbb{N},
$$

where $B_{n}$ denote the Bernoulli numbers with the convention $B_{1}=+1 / 2$. As a consequence, $\zeta$ vanishes at negative even integers, so the function $\mathcal{Z}: s \mapsto$ $\Gamma(s) \zeta(s)$ is in fact regular at these points, and the set of its poles is $\mathfrak{P}_{\mathcal{Z}}(\mathbb{C})=$ $\{1,0,-1,-3,-5, \ldots\}$. Theorem 7 therefore yields

$$
\operatorname{htr}_{P_{0}}(t) \underset{t \downarrow 0}{\sim} t^{-1}-\frac{1}{2}+\sum_{n=0}^{\infty} \frac{B_{2 n+2}}{(2 n+2) !} t^{2 n+1} .
$$

To check if this expansion is exact, by Theorem 5, we need to find an explicit bound of the form (26) for a suitable sequence $\left(R_{n}\right)$ tending monotonically to $+\infty$. We can, for instance, choose

$$
R_{0}=-\frac{3}{2}, \quad R_{1}=-\frac{1}{2}, \quad R_{2}=\frac{1}{2}, \quad R_{n}=2(n-2), \quad \text { for } n \geqslant 3,
$$

as $\mathcal{Z}$ is regular at negative even integers. Now, recall the Riemann functional equation [1, Formula (23.2.6)]:

$$
\zeta(s)=2^{s} \pi^{s-1} \sin \left(\frac{\pi s}{2}\right) \Gamma(1-s) \zeta(1-s) .
$$

By changing $s$ to $(1-s)$ we get

$$
\mathcal{Z}(s)=\Gamma(s) \zeta(s)=\frac{2^{s-1} \pi^{s} \zeta(1-s)}{\sin (\pi(1-s) / 2)} .
$$

For $s=-R_{n}+i y$ with $n \geqslant 3$ the denominator of the above expression equals

$\left.\sin \left(\pi\left(\frac{1}{2}+n-i \frac{y}{2}\right)\right)=\cos \left(\pi\left(n-i \frac{y}{2}\right)\right)=(-1)^{n} \cos \left(-i \frac{\pi y}{2}\right)\right)=(-1)^{n} \cosh \left(\frac{\pi y}{2}\right)$.

Thus, we have

$$
\left|\mathcal{Z}\left(-R_{n}+i y\right)\right|=\frac{2^{-2 n-1} \pi^{-2 n}|\zeta(2 n+1-i y)|}{\cosh \left(\frac{\pi y}{2}\right)}
$$


For any $y \in \mathbb{R}, 2 \cosh (y)>\mathrm{e}^{|y|}$ and if $x>1$ then

$$
|\zeta(x+i y)| \leqslant \sum_{k=1}^{\infty}\left|k^{-x-i y}\right|=\sum_{k=1}^{\infty} k^{-x}=\zeta(x) .
$$

Hence,

$$
|\mathcal{Z}(-2 n+i y)| \leqslant(2 \pi)^{-2 n} \zeta(2 n+1) \mathrm{e}^{-\frac{\pi|y|}{2}},
$$

what means that $\mathcal{Z}$ satisfies the assumptions of Theorem 4 with $C_{n}:=$ $(2 \pi)^{-2 n} \zeta(2 n+1)$ and a constant $\epsilon_{n}:=\pi / 2$. Now,

$$
\limsup _{n \rightarrow \infty} \sqrt[R_{n}]{C_{n} / \epsilon_{n}}=\frac{1}{2 \pi}<\infty
$$

as $\zeta(x) \rightarrow 1$ for $x \rightarrow \infty$. Thus, on the strength of Theorem 5 , we see that the expansion (41) is exact on $(0,2 \pi)$. Moreover, with the choice (42) of the sequence $\left(R_{n}\right)$, Proposition 5 applies and the expansion (41) is in fact absolutely exact on $(0,2 \pi)$.

Let us now turn to the general case of an operator $P$ with $A(n)=a(n+\alpha)$ and $B(n)=\sum_{j=0}^{\operatorname{deg} B} b_{j} n^{j}$. For any $t>0$ we have

$\operatorname{htr}_{P}(t)=\sum_{n=0}^{\infty} \sum_{j=0}^{\operatorname{deg} B} b_{j} n^{j} \mathrm{e}^{-t a(n+\alpha)}=\mathrm{e}^{-t a \alpha} \sum_{j=0}^{\operatorname{deg} B} b_{j} \sum_{n=0}^{\infty} n^{j} \mathrm{e}^{-t a n}=\mathrm{e}^{-t a \alpha} \sum_{j=0}^{\operatorname{deg} B} b_{j} \frac{d^{j}}{d t^{j}} \operatorname{htr}_{P_{0}}(a t)$.

Since $\mathbb{R}^{+} \ni t \mapsto \operatorname{htr}_{P_{0}}(t)$ is an analytic function with an absolutely exact expansion for $t \in(0,2 \pi)$, we conclude that the expansion of $\operatorname{htr}_{P}$ is absolutely exact for $(0,2 \pi / a)$.

Remark 5 Since the eigenvalues of the operator at hand grow linearly, the heat trace

$$
\operatorname{htr}_{P}(t)=\sum_{n=0}^{\infty} B(n) \mathrm{e}^{-t A(n)}
$$

associated with $P$ can be summed explicitly - for instance, $\operatorname{htr}_{P_{0}}(t)=\frac{1}{2}\left(\operatorname{coth} \frac{t}{2}-1\right)$ for any $t>0$. Hence, $\operatorname{htr}_{P}$, as a linear combination of derivatives of $\operatorname{htr}_{P_{0}}$, is actually a complex analytic function of $t$ around 0 . In particular, it admits a Laurent expansion around $t=0$ with the radius of convergence precisely equal to $2 \pi / a$ (recall that $\operatorname{coth} \frac{t}{2}$ is singular at $t=2 \pi i$ ).

We conclude that in this particular subclass the asymptotic expansion of $\operatorname{htr}_{P}(t)$ as $t \downarrow 0$ obtained from the Mellin transform is equal to the Laurent expansion of $\operatorname{htr}_{P}$ and the value of abscissa of convergence of the former precisely coincides with the value of the radius of convergence of the latter. In particular, it shows that with the estimates adopted in the statement of Theorem 5 one can obtain the maximal region of convergence of the expansion of a heat trace.

Proposition 9 has a direct geometrical application: 
Corollary 1 Let $\mathcal{D}$ be the Dirac operator acting on a spinor bundle of $S^{d}-$ the $d$-dimensional sphere with round metric. Then, the asymptotic expansion of the heat trace associated with $|\mathcal{D}|$ is exact on $(0,2 \pi)$.

Proof Recall first that on $S^{1}$ there are two possible spin structures, whereas for $S^{d}$ with $d \geqslant 2$ there is only one available [4]. In the case of the trivial spin structure on $S^{1}$ one has

$$
\lambda_{ \pm n}(\mathcal{D})= \pm n, \quad M_{ \pm n}(\mathcal{D})=1, \quad \text { for } n \in \mathbb{N},
$$

hence

$$
\lambda_{n}(|\mathcal{D}|)=n, \quad M_{n}(|\mathcal{D}|)=2-\delta_{n, 0}, \text { for } n \in \mathbb{N} .
$$

So, for $S^{1}$ with the trivial spin structure the operator $|\mathcal{D}|$ is not invertible and we have to use truncated zeta-function. From Remark 3 we get

$$
\operatorname{htr}_{|\mathcal{D}|}=1+\operatorname{htr}_{|\mathcal{D}|}^{1}=1+2 \operatorname{htr}_{P_{0}},
$$

where $P_{0}$ is as in the proof of Proposition 9.

In the case of the non-trivial spin structure, the spectrum of the Dirac operator $\mathcal{D}$ fits into the general pattern for $S^{d}[4,6,69]$ :

$$
\lambda_{ \pm n}(\mathcal{D})= \pm\left(n+\frac{d}{2}\right), \quad M_{ \pm n}(\mathcal{D})=2^{\left\lfloor\frac{d}{2}\right\rfloor}\left(\begin{array}{c}
n+d-1 \\
d-1
\end{array}\right), \quad \text { for } n \in \mathbb{N} .
$$

Hence, the operator $|\mathcal{D}|$ with

$$
\lambda_{n}(|\mathcal{D}|)=n+\frac{d}{2}, \quad M_{n}(|\mathcal{D}|)=2^{\left\lfloor\frac{d}{2}\right\rfloor+1}\left(\begin{array}{c}
n+d-1 \\
d-1
\end{array}\right), \quad \text { for } n \in \mathbb{N},
$$

meets the conditions of Proposition 9 with $a=1, \alpha=\frac{d}{2}$ and $B(n)=M_{n}$ and the assertion follows.

\subsubsection{Almost Exact Expansions}

In this subsection we turn to the case of almost exact (but not exact) expansions of heat traces associated with operators of polynomial spectrum.

Proposition 10 Let $P$ be an operator with eigenvalues $\lambda_{n}=A(n)$ and multiplicities $M_{n}=B(n)$ for $n \in \mathbb{N}$. If $A(n)=a(n+\alpha)^{2 k}+\beta$ for some $a \in \mathbb{R}^{+}, \alpha \in \frac{1}{2} \mathbb{N}, \beta \in \mathbb{R}$, $k \in \mathbb{N}^{+}$and $B(n)=\sum_{j=0}^{(\operatorname{deg} B) / 2} \widetilde{b}_{j}(n+\alpha)^{2 j}$ with $\operatorname{deg} B$ even, then the asymptotic expansion (40) is almost exact for all $t>0$, but not exact.

Proof At first let us note that with $P=P^{\prime}+\beta$ we have

$$
\operatorname{htr}_{P}(t)=\mathrm{e}^{-t \beta} \operatorname{htr}_{P^{\prime}}(t) .
$$

Thus, it is sufficient to consider the case $\beta=0$. 
Moreover, we can assume that $\alpha=\frac{1}{2}$ or $\alpha=0$. Indeed, let us denote by $\tilde{\alpha}=$ $\alpha-\lfloor\alpha\rfloor$ the fractional part of $\alpha$, then we have

$$
\begin{aligned}
\operatorname{htr}_{P^{\prime}}(t) & =\sum_{n=0}^{\infty} B(n) \mathrm{e}^{-t A(n)}=\sum_{j=0}^{(\operatorname{deg} B) / 2} \widetilde{b}_{j} \sum_{n=0}^{\infty}(n+\alpha)^{2 j} \mathrm{e}^{-t a(n+\alpha)^{2 k}}= \\
& =\sum_{j=0}^{(\operatorname{deg} B) / 2} \widetilde{b}_{j} \sum_{n=0}^{\infty}(n+\lfloor\alpha\rfloor+\widetilde{\alpha})^{2 j} \mathrm{e}^{-t a(n+\lfloor\alpha\rfloor+\widetilde{\alpha})^{2 k}}= \\
& =\sum_{j=0}^{(\operatorname{deg} B) / 2} \widetilde{b}_{j} \sum_{n=\lfloor\alpha\rfloor}^{\infty}(n+\widetilde{\alpha})^{2 j} \mathrm{e}^{-t a(n+\widetilde{\alpha})^{2 k}}= \\
& =\sum_{j=0}^{(\operatorname{deg} B) / 2} \widetilde{b}_{j} \sum_{n=0}^{\infty}(n+\widetilde{\alpha})^{2 j} \mathrm{e}^{-t a(n+\widetilde{\alpha})^{2 k}}-\sum_{j=0}^{(\operatorname{deg} B) / 2} \widetilde{b}_{j} \sum_{n=0}^{\lfloor\alpha\rfloor-1}(n+\widetilde{\alpha})^{2 j} \mathrm{e}^{-t a(n+\widetilde{\alpha})^{2 k}},
\end{aligned}
$$

and the second term is regular at $t=0$.

Let us denote by $P^{\prime \prime}$ the operator with $\lambda_{n}\left(P^{\prime \prime}\right)=a(n+\widetilde{\alpha})^{2 k}$ and $M_{n}\left(P^{\prime \prime}\right)=$ $M_{n}(P)$. For $\mathfrak{R}(s)>(1+\operatorname{deg} B) /(\operatorname{deg} A)$ we have

$$
\zeta_{P^{\prime \prime}}(s)=\sum_{n=0}^{\infty} \sum_{j=0}^{(\operatorname{deg} B) / 2} \tilde{b}_{j} a^{-s}(n+\widetilde{\alpha})^{2 j-2 k s}=a^{-s} \sum_{j=0}^{(\operatorname{deg} B) / 2} \tilde{b}_{j} \zeta_{H}(2 k s-2 j, \widetilde{\alpha}),
$$

where $\zeta_{H}$ is the Hurwitz zeta-function. This formula provides a meromorphic extension of $\zeta_{P^{\prime \prime}}$ to the whole complex plane. Since the Hurwitz zeta-function has a single simple pole at 1 for any $\widetilde{\alpha} \in \mathbb{C} \backslash-\mathbb{N}$, we have $\mathfrak{P}_{\zeta_{P^{\prime \prime}}}(\mathbb{C})=\frac{1}{2 k}\{1,3, \ldots, \operatorname{deg} B+1\}$. What is more, for $s=-n$ with $n \in \mathbb{N}$ we have

$$
\zeta_{P^{\prime \prime}}(-n)=a^{n} \sum_{j=0}^{(\operatorname{deg} B) / 2} \tilde{b}_{j} \zeta_{H}(-2 k n-2 j, \widetilde{\alpha})=-a^{n} \sum_{j=0}^{(\operatorname{deg} B) / 2} \tilde{b}_{j} \frac{B_{2 k n+2 j+1}(\widetilde{\alpha})}{2 k n+2 j+1},
$$

where $B_{n}$ denotes the Bernoulli polynomial of degree $n$. But if $\widetilde{\alpha}=0$ or $\widetilde{\alpha}=\frac{1}{2}$ then $B_{2 n+1}(\widetilde{\alpha})=0\left[1\right.$, Section 23], hence for any $n \in \mathbb{N}^{+} \zeta_{P^{\prime \prime}}(-n)=0$. So if $\mathcal{Z}(s)=\Gamma(s) \zeta_{P^{\prime \prime}}(s)$ as usually, we have $\mathfrak{P}_{\mathcal{Z}}(\mathbb{C})=\frac{1}{2 k}\{0,1,3, \ldots, \operatorname{deg} B+1\}$. Thus, on the strength of Proposition 6 and formula (40) we finally conclude that for all $t>0$

$$
\begin{aligned}
& \operatorname{htr}_{P}(t)=\mathrm{e}^{-t \beta} \sum_{j=0}^{(\operatorname{deg} B) / 2} \tilde{b}_{j} \Gamma\left(\frac{1+2 j}{2 k}\right)(a t)^{-\frac{1+2 j}{2 k}}-\mathrm{e}^{-t \beta} \widetilde{b}_{0}\left(\widetilde{\alpha}-\frac{1}{2}\right)+ \\
&-\mathrm{e}^{-t \beta} \sum_{j=0}^{(\operatorname{deg} B) / 2} \tilde{b}_{j} \sum_{n=0}^{\lfloor\alpha\rfloor-1}(n+\widetilde{\alpha})^{2 j} \mathrm{e}^{-t a(n+\widetilde{\alpha})^{2 k}}+F_{\infty}(t),
\end{aligned}
$$

where $F_{\infty}(t) \neq 0$ has a null Taylor expansion at $t=0$. 
In some cases, one could be able to compute the remainder function $F_{\infty}$ explicitly.

Example 2 Let $P$ be an operator with $\lambda_{n}(P)=n^{2}$ and $M_{n}=1$ for $n \in \mathbb{N}$, then

$$
\operatorname{htr}_{P}(t)=\sum_{n=0}^{\infty} \mathrm{e}^{-t n^{2}}=\frac{1}{2}\left(\theta_{3}\left(0 ; \mathrm{e}^{-t}\right)-1\right),
$$

with $\theta_{3}$ being the Jacobi theta function defined as

$$
\theta_{3}(z ; q):=\sum_{n=-\infty}^{\infty} q^{n^{2}} \mathrm{e}^{2 n i z} .
$$

For such an operator one can compute explicitly (see [30] and also [28]):

$$
F_{\infty}(t)=\sqrt{\frac{\pi}{t}} \frac{1}{2}\left(-1+\theta_{3}\left(0 ; \mathrm{e}^{-\pi^{2} / t}\right)\right) .
$$

Hence, formula (44) turns out to be the famous Jacobi identity [30, (3.13)]. This example has been analysed in details in [30] and has direct applications to quantum physics.

Therefore, one can regard the formula (44) as a generalisation of the Jacobi identity for special functions defined by $\operatorname{htr}_{P}(t)$ with $P$ as in Proposition 10 .

Proposition 10 again has a direct geometrical application.

Corollary 2 Let $\mathcal{D}$ be the Dirac operator acting on a spinor bundle of $S^{d}-$ the $d$ dimensional sphere with round metric. If $d$ is odd, then the asymptotic expansion of the heat trace associated with $\mathcal{D}^{2 k}$ with $k \in \mathbb{N}^{+}$is almost exact for all $t>0$, but not exact.

Proof To apply Proposition 10 we need to show that $M_{n}\left(\mathcal{D}^{2 k}\right)$ can be written as a polynomial in $\lambda_{n}\left(\mathcal{D}^{2}\right)$. For trivial spin structure on $S^{1}$ it is obvious, however we need to resort again to the truncated zeta-function, as in Corollary 1.

In all other cases, by formula (43) we have $\lambda_{n}\left(\mathcal{D}^{2 k}\right)=\left(n+\frac{d}{2}\right)^{2 k}$. Note that

$$
\begin{aligned}
M_{n}\left(\mathcal{D}^{2 k}\right) & =M_{n}(|\mathcal{D}|) \\
& =2^{\left\lfloor\frac{d}{2}\right\rfloor+1}\left(\lambda_{n}(|\mathcal{D}|)+\frac{d}{2}-1\right)\left(\lambda_{n}(|\mathcal{D}|)+\frac{d}{2}-2\right) \cdots\left(\lambda_{n}(|\mathcal{D}|)-\frac{d}{2}+2\right)\left(\lambda_{n}(|\mathcal{D}|)-\frac{d}{2}+1\right) .
\end{aligned}
$$

So if $d$ is odd, then

$$
M_{n}\left(\mathcal{D}^{2 k}\right)=2^{\left\lfloor\frac{d}{2}\right\rfloor+1}\left(\lambda_{n}(|\mathcal{D}|)^{2}-\left(\frac{d}{2}-1\right)^{2}\right)\left(\lambda_{n}(|\mathcal{D}|)^{2}-\left(\frac{d}{2}-2\right)^{2}\right) \cdots\left(\lambda_{n}(|\mathcal{D}|)^{2}-\left(\frac{1}{2}\right)^{2}\right)
$$

Hence, $M_{n}\left(\mathcal{D}^{2 k}\right)$ can indeed be written as a polynomial in $\lambda_{n}(|\mathcal{D}|)^{2}=\lambda_{n}\left(\mathcal{D}^{2}\right)$. 
The comparison of Corollaries 1 and 2 shows a sharp contrast between the heat traces associated with the operators $|\mathcal{D}|$ and $\mathcal{D}^{2}$ for odd-dimensional spheres. For the even-dimensional ones the discrepancy is even more dramatic, as we shall now see.

\subsubsection{Divergent Expansions}

For a generic operator of polynomial spectrum with eigenvalues growing at least quadratically one expects the associated heat trace expansion to be only an asymptotic one. In particular, we have the following result:

Proposition 11 Let $\mathcal{D}$ be the Dirac operator acting on a spinor bundle of $S^{d}-$ the $d$-dimensional sphere with round metric. If $d$ is even, then the asymptotic expansion of the heat trace associated with $\mathcal{D}^{2}$ is only asymptotic.

Proof If $d$ is even, formula (46) yields

$$
\begin{aligned}
M_{n}\left(\mathcal{D}^{2}\right) & =2^{\left\lfloor\frac{d}{2}\right\rfloor+1}\left(\lambda_{n}(|\mathcal{D}|)^{2}-\left(\frac{d}{2}-1\right)^{2}\right)\left(\lambda_{n}(|\mathcal{D}|)^{2}-\left(\frac{d}{2}-2\right)^{2}\right) \cdots\left(\lambda_{n}(|\mathcal{D}|)^{2}-1^{2}\right) \lambda_{n}(|\mathcal{D}|) \\
& =: \sum_{m=0}^{d-1} c_{m} \lambda_{n}(|\mathcal{D}|)^{m}
\end{aligned}
$$

with $c_{m}=0$ for $m$ even. Moreover,

$$
\operatorname{sign} c_{d-1-2 q}=(-1)^{q}, \forall q=0, \ldots, \frac{d}{2}-1 .
$$

The zeta-function associated with the operator $P=\mathcal{D}^{2}$ on $S^{d}$ reads

$$
\zeta_{P}(s)=\sum_{n=0}^{\infty} M_{n}\left(\mathcal{D}^{k}\right) \lambda_{n}(|\mathcal{D}|)^{-2 s}=\sum_{m=0}^{d-1} c_{m} \sum_{n=0}^{\infty}\left(n+\frac{d}{2}\right)^{-2 s+m}=\sum_{m=0}^{d-1} c_{m} \zeta_{H}\left(2 s-m, \frac{d}{2}\right),
$$

where $\zeta_{H}$ is the Hurwitz zeta-function. It turns out, that the latter can actually be replaced by the Riemann zeta-function. Indeed, let us note that

$$
\zeta_{H}\left(s, \frac{d}{2}\right)=\sum_{n=0}^{\infty}\left(n+\frac{d}{2}\right)^{-s}=\sum_{n=1}^{\infty} n^{-s}-\sum_{n=1}^{d / 2-1} n^{-s}=\zeta(s)-F_{d}(s),
$$

with $F_{d}(s):=\sum_{n=1}^{d / 2-1} n^{-s}$ for $d \geqslant 4$ and $F_{2}(s)=0$. Hence,

$$
\zeta_{P}(s)=\sum_{m=0}^{d-1} c_{m} \zeta(2 s-m)-\sum_{m=0}^{d-1} c_{m} F_{d}(2 s-m)
$$


Let us investigate the second term on the RHS of the above equality. For any $s \in \mathbb{C}$,

$$
\sum_{m=0}^{d-1} c_{m} F_{d}(2 s-m)=\sum_{n=1}^{d / 2-1} n^{-2 s} \sum_{m=0}^{d-1} c_{m} n^{m}=\sum_{n=1}^{d / 2-1} n^{-2 s} B\left(n-\frac{d}{2}\right),
$$

where $B$ is the polynomial defining the multiplicites of $\mathcal{D}^{2}$, i.e. $M_{n}\left(\mathcal{D}^{2}\right)=B(n)$ for all $n \in \mathbb{N}$. But, since $B(n)=2^{\left\lfloor\frac{d}{2}\right\rfloor+1}\left(\begin{array}{c}n+d-1 \\ d-1\end{array}\right)$ by (43) and $d$ is even, we have $B\left(n-\frac{d}{2}\right)=0$ for every $n=1, \ldots, \frac{d}{2}-1$. Thus,

$$
\zeta_{P}(s)=\sum_{m=0}^{d-1} c_{m} \zeta(2 s-m)
$$

Now, Theorem 7 yields the following asymptotic expansion:

$$
\begin{aligned}
\operatorname{htr}_{P}(t) \underset{t \downarrow 0}{\sim} \sum_{k=0}^{\infty} \operatorname{Res}_{s=(d-k) / 2} \Gamma(s) \zeta_{P}(s) t^{(k-d) / 2} \\
\quad=\sum_{k=0}^{d-1} \Gamma\left(\frac{d-k}{2}\right) c_{d-k-1} t^{(k-d) / 2}+\sum_{p=0}^{\infty} \frac{(-1)^{p}}{p !} \sum_{m=0}^{d-1} c_{m} \zeta(-2 p-m) t^{p} .
\end{aligned}
$$

Let us investigate the convergence of the series

$$
\sum_{p=0}^{\infty} d_{p} t^{p}, \quad \text { with } \quad d_{p}=\frac{(-1)^{p}}{p !} \sum_{m=0}^{d-1} c_{m} \zeta(-2 p-m) .
$$

Recall that [1, (23.2.15)]

$$
\zeta(-2 p-m)=-\frac{B_{2 p+m+1}}{2 p+m+1},
$$

where $B_{n}$ are the Bernoulli numbers.

Since $c_{m}=0$ for even $m$, therefore in the definition of $d_{p}$ the only non-vanishing terms are those with $2 p+m+1$ even. Moreover, we have

$$
\operatorname{sign} B_{2 n}=(-1)^{n+1} \text {, }
$$

and by (46)

$$
\operatorname{sign} c_{m}=(-1)^{\frac{d-1-m}{2}} \text {, }
$$

thus

$$
\operatorname{sign} c_{m} B_{2 p+m+1}=(-1)^{\frac{d-1-m}{2}}(-1)^{\frac{2 p+m+1}{2}+1}=(-1)^{\frac{d}{2}+p+1} .
$$

Therefore,

$$
\operatorname{sign} d_{p}=(-1)^{\frac{d}{2}}
$$

so all of the terms in series (48) over $p$ have the same sign. Moreover, since sign $c_{m} B_{2 p+m+1}$ does not depend on $m$ it is sufficient to study the behaviour of $c_{m} \zeta(-2 p-m) /(p !)$ as $p$ grows, for a fixed $m$. 
We have [1, (23.1.15)]

$$
(-1)^{n+1} B_{2 n}>\frac{2(2 n) !}{(2 \pi)^{n}},
$$

so that

$$
c_{m} \frac{\zeta(-2 p-m)}{p !}=c_{m} \frac{B_{2 p+m+1}}{p !(2 p+m+1)}>\frac{2\left|c_{m}\right|}{(2 \pi)^{(m+1) / 2}} \frac{(2 p+m) !}{(2 \pi)^{p} p !} \underset{p \rightarrow \infty}{\longrightarrow} \infty .
$$

Hence, the asymptotic series in (47) diverges for any $t>0$.

The lesson from the example of spheres is that if one is interested in the convergence properties of heat trace expansion one should work with $|\mathcal{D}|$, which is of the first order, rather than with $\mathcal{D}^{2}$, despite the fact that the former is not a differential operator, but only a pseudodifferential one.

\subsection{Operators of Exponential Spectrum}

In this section we consider the class of operators of exponential spectrum, i.e. the ones with the spectrum $\sigma(P)=\left(q^{-n}\right)_{n \in \mathbb{N}}$ for some $0<q<1$. We shall also assume that the multiplicities are given by a polynomial. This type of operators appears naturally in the context of quantum groups [50, 51] and their homogeneous spaces [27, 29, 60] (see also [52] and [35, Example 12]), as well as in the framework of fractal spaces [11-13, 41, 42].

Proposition 12 Let $P$ be an operator with $\lambda_{n}=q^{-n}$ for some $0<q<1$ and $M_{n}=p(n)$ for some polynomial $p$ of degree $m$. Then, the asymptotic expansion of the heat trace $\operatorname{htr}_{P}$ is absolutely exact for all $t>0$ and can be expressed as

$$
\operatorname{htr}_{P}(t)=\frac{\widetilde{p}(1)}{(m+1) !}\left(-\log _{q} t\right)^{m+1}+\sum_{j=0}^{m}\left(r_{j}+G_{j}\left(\log _{q} t\right)\right)\left(\log _{q} t\right)^{j}+\sum_{n=1}^{\infty} \frac{\widetilde{p}\left(q^{-n}\right)}{\left(1-q^{-n}\right)^{m+1}} \frac{(-t)^{n}}{n !}
$$

$\log _{q} t=\frac{\log t}{\log q}, \tilde{p}$ is a polynomial of degree $m, r_{i}$ are constants (with respect to $t$ ) and $G_{i}$ are Fourier series completely determined by $q$ and the polynomial $p$.

Proof We start with the analysis of the zeta-function. For $\mathfrak{R}(s)>0$ we have

$$
\zeta_{P}(s)=\sum_{n=0}^{\infty} p(n) q^{n s}=\sum_{j=0}^{m} p_{j} \sum_{n=0}^{\infty} n^{j} q^{n s}=: \frac{\tilde{p}\left(q^{s}\right)}{\left(1-q^{s}\right)^{m+1}} .
$$

The polynomial $\tilde{p}$ is completely determined by $p$ via the formula

$$
\sum_{n=0}^{\infty} n^{j} q^{n s}=\mathrm{Li}_{-j}\left(q^{s}\right)=\frac{1}{\left(1-q^{s}\right)^{j+1}} \sum_{k=0}^{j-1}\left\langle\begin{array}{l}
j \\
k
\end{array}\right) q^{s(j-k)}
$$


where $\mathrm{Li}$ is the polylogarithm (Jonquière's) function [49] and $\left\langle\begin{array}{l}j \\ k\end{array}\right\rangle$ stand for Eulerian numbers of the first kind [14]. From the formula (50) we also deduce that $\widetilde{p}(1)=p_{m} \cdot m ! \neq 0$. Indeed,

$$
\tilde{p}\left(q^{s}\right)=\sum_{j=0}^{m} p_{j}\left(1-q^{s}\right)^{m-j} \sum_{k=0}^{j-1}\left\langle\begin{array}{l}
j \\
k
\end{array}\right) q^{s(j-k)}
$$

and, using the summation formula for Eulerian numbers [14, p. 242],

$$
\tilde{p}(1)=p_{m} \sum_{k=0}^{m-1}\left\langle\begin{array}{l}
m \\
k
\end{array}\right\rangle=p_{m} \cdot m ! .
$$

Therefore, we conclude that the function $\mathcal{Z}: s \mapsto \Gamma(s) \zeta_{P}(s)$ has a meromorphic extension to the whole complex plane with:

- first order poles at $s \in-\mathbb{N}^{+}$,

- $\quad m+1$ order poles at $s \in \frac{2 \pi i}{\log q} \mathbb{Z}^{*}$,

- $\quad m+2$ order pole at $s=0$.

To see that the assumptions of Theorem 4 are met let us choose $R_{n}=n+\frac{1}{2}$ and $y_{k}^{(n)}=\frac{2 \pi}{\log q}\left(k+\frac{1}{2}\right)$. Let us also denote by $\hat{p}$ the polynomial $\hat{p}(x)=\sum_{i=0}^{m}\left|\tilde{p}_{i}\right| x^{i}$.

On the horizontal lines of the contour integration we have

$$
\left|\zeta_{P}\left(x+i y_{k}^{(n)}\right)\right|=\frac{\left|\widetilde{p}\left(q^{x+i y_{k}^{(n)}}\right)\right|}{\left|1-q^{x+i y_{k}^{(n)}}\right|^{m+1}}=\frac{\left|\tilde{p}\left(q^{x+i y_{k}^{(n)}}\right)\right|}{\left(1+q^{x}\right)^{m+1}} \leqslant \frac{\hat{p}\left(q^{x}\right)}{\left(1+q^{x}\right)^{m+1}}
$$

and $\Gamma$ decays exponentially on verticals [63, (2.1.21)], hence Assumption 4 of Theorem 4 is fulfilled. Similarly, on the vertical lines of integration we have

$$
\left|\zeta_{P}\left(-R_{n}+i y\right)\right|=\frac{\left|\widetilde{p}\left(q^{-R_{n}+i y}\right)\right|}{\left|1-q^{-R_{n}+i y}\right|^{m+1}} \leqslant \frac{\hat{p}\left(q^{-R_{n}}\right)}{\left|1-q^{-R_{n}}\right|^{m+1}} .
$$

To show that we have an exact expansion of $\operatorname{htr}_{P}$ valid for any $t>0$ we need to estimate the Gamma function more precisely. The Euler reflection formula [63, (2.1.20)] together with the estimate (see [29, Formula (43)])

$$
|\Gamma(x+i y)|^{-1} \leqslant(2 \pi)^{-1 / 2} \mathrm{e}^{x+|y||\arg (x+i y)|}\left(x^{2}+y^{2}\right)^{-x / 2+1 / 4}, \quad \text { for } x>0, y \in \mathbb{R}
$$

gives

$$
\begin{aligned}
\left|\Gamma\left(-R_{n}+i y\right)\right| & \leqslant \sqrt{\frac{\pi}{2}} \frac{1}{\cosh (\pi y)} \mathrm{e}^{1+R_{n}+|y||1+R-i y|}\left(\left(1+R_{n}\right)^{2}+y^{2}\right)^{-R_{n} / 2-1 / 4} \\
& \leqslant \sqrt{\frac{\pi}{2}} \mathrm{e}^{1+R_{n}} R_{n}^{-R_{n}-1 / 2} \frac{1}{\cosh (\pi y)} \mathrm{e}^{|y||1+R-i y|} \\
& \leqslant \sqrt{2 \pi} \mathrm{e}^{1+R_{n}} R_{n}^{-R_{n}-1 / 2} \frac{\mathrm{e}^{\frac{\pi}{2}|y|}}{\mathrm{e}^{\pi y}+\mathrm{e}^{-\pi y}} \\
& \leqslant \sqrt{2 \pi} \mathrm{e}^{1+R_{n}} R_{n}^{-R_{n}-1 / 2} \mathrm{e}^{-\frac{\pi}{2}|y|}
\end{aligned}
$$


The above result together with (51) yields the following constants in Assumption 3 of Theorem 5:

$$
\epsilon_{n}=\frac{\pi}{2}, \quad C_{n}=\sqrt{2 \pi} \mathrm{e}^{3 / 2} \frac{\mathrm{e}^{n} \hat{p}\left(q^{-n-1 / 2}\right)}{\left|1-q^{-n-1 / 2}\right|}\left(n+\frac{1}{2}\right)^{-n} .
$$

Therefore, Theorem 5 applies and

$$
\limsup _{n \rightarrow \infty} \sqrt[R_{n}]{C_{n} / \epsilon_{n}}=0, \quad \text { for any } 0<q<1
$$

yielding $T=\infty$. Moreover, since $R_{n}=n+\frac{1}{2}$, Proposition 5 applies and the expansion is absolutely exact.

The formula (49) results from a direct calculation of the residues.

This result is somewhat surprising at the first sight. Clearly, $\operatorname{htr}_{P}(t)$ decays exponentially as $t$ grows to infinity, whereas the RHS of (49) seems to diverge to infinity because of the $\log t$ terms. However, it turns out that the sum over $n$ in the RHS of (49) compensates for large $t$ the divergent terms $(\log t)^{j}$, the constant terms and the oscillatory part to yield an exponential decay (compare [35, Example 12] and [29, Section 4.1]).

It is interesting to note that if $P$ is an operator of exponential spectrum, then so is $P^{r}$ for any $r \in \mathbb{R}_{+}$(compare also [29, Section 4.4]). Moreover, given the exact expansion of the heat trace (49), one immediately obtains that for $\operatorname{htr}_{P} r$ simply by changing $q \rightsquigarrow q^{r}$.

\section{Outlook}

We start the concluding section of this paper with an exploration of the limitations of our general theorems. Having in mind the exactness result of the previous section one could naively expect that the faster the eigenvalues of a positive operator grow, the better the convergence properties of the associated heat trace expansion are. However, as we shall show below the exponential growth of eigenvalues establishes in fact a limit of applicability of the inverse Mellin transform.

Proposition 13 Let $P$ be such that $M_{n}=\mathcal{O}_{\infty}\left(n^{b}\right)$ for some $b \in \mathbb{R}^{+}$. If

$$
\lim _{n \rightarrow \infty} \frac{\lambda_{n+1}}{\lambda_{n}}=+\infty,
$$

then the function $\zeta_{P}$ is holomorphic for $\mathfrak{R}(s)>0$, but does not admit a meromorphic continuation through $\mathfrak{R}(s)=0$.

Proof The general Dirichlet series defining the zeta-function associated with $P$ reads

$$
\zeta_{P}(s)=\sum_{n=0}^{\infty} M_{n} \lambda_{n}^{-s}=\sum_{n=0}^{\infty} M_{n} \mathrm{e}^{-s \log \lambda_{n}} .
$$


We note that (53) implies that $\lambda_{n}=\mathcal{O}_{\infty}\left(\mathrm{e}^{g(n)}\right)$ with $g(n) / n \rightarrow+\infty$. Moreover, the assumption on the power-like growth of multiplicites assures that $\zeta_{P}(s)$ is convergent for $\Re(s) \geqslant 0$ - see [23,45] and references therein.

Under the assumption (53), the series (54) is a lacunary Dirichlet series [45]:

$$
\zeta_{P}(s)=\sum_{n=0}^{\infty} a_{n} z^{\mu_{n}},
$$

with $z=\mathrm{e}^{-s}, \mu_{n}=\log \lambda_{n}$ and $a_{n}=M_{n}$. Then, classical results [55] (see also [33], [56, Theorem 1], [45] and [23]) show that the vertical line $\mathfrak{R}(s)=0$ is a natural boundary of analyticity for $\zeta_{P}(s)$. The latter means that the poles of $\zeta_{P}$ are dense on the imaginary axis and therefore, $\zeta_{P}$ cannot be extended to the left complex halfplane.

We shall call the operators satisfying the assumptions of Proposition 13 lacunary operators. Proposition 13 does not imply directly that $\operatorname{htr}_{P}(t)$ does not have an asymptotic expansion as $t \downarrow 0$ for lacunary operators - in particular, both $\operatorname{htr}_{P}$ and $\zeta_{P}$ are well-defined. It just states that the technique of the inverse Mellin transform developed in this paper does not apply in this case. For an a example of a lacunary operator in the realm of non-classical pseudodifferential operators see [64]. ${ }^{2}$

Can anything be said about the small $t$ behaviour of heat traces associated with lacunary operators? The answer is positive and can be deduced from the following Tauberian Theorem due to Hardy and Littlewood (known also under the name of Karamata Theorem).

Theorem 8 ([34] Theorem XIII.5.2) Let $G: \mathbb{R}^{+} \rightarrow \mathbb{R}$ be a function of bounded variation (see [73, Chapter 1]) and such that the following Riemann-Stieltjes integral

$$
\omega(t)=\int_{0}^{\infty} \mathrm{e}^{-t \lambda} d G(\lambda),
$$

converges for $t>0$. Then, the following are equivalent:

$$
G(\lambda) \approx \lambda^{L} F(\lambda), \text { as } \lambda \rightarrow \infty,
$$

and

$$
\omega(t) \approx t^{-L} \Gamma(L+1) F\left(t^{-1}\right), \quad \text { as } t \downarrow 0,
$$

where $F$ is a slowly varying function, i.e $F(\tau x) / F(\tau) \rightarrow 1$ as $\tau \rightarrow \infty$ for every $x>0$.

As a direct application of Theorem 8 we obtain the following result:

Corollary 3 Let $P$ be a positive operator such that $\zeta_{P}$ has a finite abscissa of convergence $L$. If

$$
N(\lambda) \approx \lambda^{L} F(\lambda)
$$

\footnotetext{
${ }^{2}$ We thank Bruno Iochum for pointing out this reference to us.
} 
with $F$ slowly varying, then

$$
\operatorname{htr}_{P}(t) \approx \Gamma(L+1) t^{-L} F\left(t^{-1}\right), \quad \text { as } t \downarrow 0 .
$$

Proof The function $N$, being a step function, is of bounded variation [73, Chapter 1]. Moreover, since $\zeta_{P}$ has a finite abscissa of convergence, $\operatorname{htr}_{P}$ is well-defined and thus

$$
\operatorname{htr}_{P}(t)=\sum_{n=0}^{\infty} M_{n} \mathrm{e}^{-t \lambda_{n}}=\int_{0}^{\infty} \mathrm{e}^{-t \lambda} d N(\lambda)
$$

for all $t>0$. Proposition 2 implies that for large $\lambda$ we have $N(\lambda) \approx \lambda^{L} F(\lambda)$, with $F(\lambda)=\mathcal{O}_{\infty}\left(\lambda^{\delta}\right)$ for every $\delta>0$. If moreover, $F$ is slowly varying, then Theorem 8 applies and the conclusion follows.

Corollary 3 applies also to lacunary operators and one can use it to detect the leading behaviour of $\operatorname{htr}_{P}$ as $t \downarrow 0$.

Example 3 Let us consider a lacunary operator $P$ with $\lambda_{n}=\mathrm{e}^{n^{2}}$ and no degeneracy (i.e. $M_{n} \equiv 1$ ). Its zeta-function reads

$$
\zeta_{P}(s)=\sum_{n=0}^{\infty} \mathrm{e}^{-s n^{2}}=\frac{1}{2}\left(\theta_{3}\left(0 ; \mathrm{e}^{-s}\right)+1\right), \quad \text { for } \mathfrak{R}(s)>0,
$$

where $\theta_{3}$ is the Jacobi theta function we met in Example 2, but now playing the role of the zeta-function. It is a lacunary function and does not admit a meromorphic continuation to the left complex half-plane.

On the other hand, since

$$
N(\lambda)=\sum_{\left\{n: \mathrm{e}^{n^{2}} \leqslant \lambda\right\}} 1 \approx \sqrt{\log \lambda}
$$

and the function $\sqrt{\log }$ is slowly varying, Theorem 8 implies

$$
\operatorname{htr}_{P}(t)=\sum_{n=0}^{\infty} \mathrm{e}^{-t \mathrm{e}^{n^{2}}} \approx \sqrt{-\log t}, \quad \text { as } t \downarrow 0 .
$$

However, the assumption of $N$ being a regularly varying function is a non-trivial one and puts limitations on the usefulness of Theorem 8 .

Example 4 Let $P$ be an operator with $\lambda_{n}(P)=2^{n}$ and $M_{n}(P)=2^{n}$. Then,

$$
N(\lambda)=\sum_{n: 2^{n} \leqslant \lambda} 2^{n}=\sum_{n=0}^{\left\lfloor\log _{2} \lambda\right\rfloor} 2^{n}=2^{\left\lfloor\log _{2} \lambda\right\rfloor+1}-1 \approx \lambda \cdot F(\lambda),
$$

but $F(\lambda):=2^{\left\lfloor\log _{2} \lambda\right\rfloor+1} \lambda^{-1}$ is not slowly varying. Indeed, the limit $\lim _{\lambda \rightarrow \infty} F(x \lambda) / F(\lambda)$ exists (and is equal to 1 ) only if $x=2^{m}$ with $m \in \mathbb{N}$. 
On the other hand,

$$
\zeta_{P}(s)=\sum_{n=0}^{\infty} 2^{n} 2^{-s n}=\frac{1}{1-2^{s-1}} .
$$

The results of Section 4.2 can be easily adapted to this setting yielding the following absolutely exact expansion

$$
\operatorname{htr}_{P}(t)=-\frac{t^{-1}}{\log 2} \sum_{k \in \mathbb{Z}} \Gamma\left(1-\frac{2 \pi i k}{\log 2}\right) t^{\frac{2 \pi i k}{\log 2}}+\sum_{n=0}^{\infty} \frac{(-1)^{n}}{n !} \frac{t^{n}}{1-2^{-n-1}},
$$

which is valid for all $t>0$. Note that the leading term of $\operatorname{htr}_{P}$ is of the form $t^{-1} G(t)$, where $G$ is oscillating and thus not slowly varying.

We have seen that the spectral growth function (3) of an operator is a primary quantity that allows us to determine whether an asymptotic expansion of the associated heat trace can be obtained via the inverse Mellin transform. Proposition 1 establishes an upper bound on the spectral growth of $P$, whereas Proposition 13 gives a lower one. One might therefore expect that for the intermediate values of growth rates, the inverse Mellin transform technique guarantees the existence of an asymptotic expansion of heat traces. However, this is not the case as we shall show below.

Proposition 14 Let $P$ be an operator such that its spectral growth function satisfies

$$
N(\lambda) \approx(\log \lambda)^{a}, \quad \text { as } \lambda \rightarrow \infty,
$$

for some a $\in \mathbb{R}^{+}$. If $a \notin \mathbb{N}$, then $\zeta_{P}$ has an abscissa of convergence $L=0$, but is not meromorphic around $s=0$.

Proof Proposition 2 implies that $\zeta_{P}$ is well-defined with an abscissa of convergence $L=0$. To see that $\zeta_{P}$ is not meromorphic around $s=0$ we invoke the HardyLittlewood Tauberian Theorem 8 once again.

With $M \in \mathbb{N}$ such that $\lambda_{M} \leqslant 1$ and $\lambda_{M+1}>1$, we write the zeta-function $\zeta_{P}$ on $\mathbb{R}^{+}$as Riemann-Stieltjes integral (compare [65, Section 13.3])

$$
\zeta_{P}(s)=\int_{0}^{\infty} \lambda^{-s} d N(\lambda), \quad \hat{\zeta}_{P}^{M}(s)=\int_{1}^{\infty} \lambda^{-s} d N(\lambda) .
$$

On the strength of [73, Theorem 11a] we can change variables in the RiemannStieltjes integral and rewrite

$$
\hat{\zeta}_{P}^{M}=\int_{1}^{\infty} \mathrm{e}^{-s \log \lambda} d N(\lambda)=\int_{0}^{\infty} \mathrm{e}^{-s \mu} d \tilde{N}(\mu)
$$

for $s>0$, with $\widetilde{N}(\mu)=\sum_{n: \log \lambda_{n} \leqslant \mu} M_{n}$, which is of bounded variation on $\mathbb{R}_{+}$. Assumption (55) implies

$$
\tilde{N}(\mu) \approx \mu^{a}, \quad \text { as } \mu \rightarrow \infty,
$$


thus, by Theorem 8 , we have

$$
\hat{\zeta}_{P}^{M}(s) \approx s^{-a} \Gamma(a+1), \quad \text { as } s \downarrow 0 .
$$

Therefore, if an extension of $\hat{\zeta}_{P}^{M}$ to $\mathfrak{R}(s) \leqslant 0$ exists at all, then the point $s=0$ is not a pole unless $a \in \mathbb{N}^{+}$. Since $\hat{\zeta}_{P}^{M}$ and $\zeta_{P}$ differ by an entire function, the same conclusion holds for $\zeta_{P}$.

Let us stress that Proposition 14 has a "no-go" character only. Having $a \in \mathbb{N}^{+}$does not imply that $\zeta_{P}$ can be extended to the left complex half-plane in a meromorphic way.

Proposition 14 is in accordance with Proposition 13, but it also provides examples of pathological operators, which are not lacunary.

Example 5 Consider an operator $P$ with $\lambda_{n}=\mathrm{e}^{n^{2 / 3}}$ and no degeneracy (i.e. $M_{n} \equiv 1$ ). Although its eigenvalues grow slower than exponentially, the zeta-function $\zeta_{P}$ cannot be meromorphic around $s=0$, as

$$
\zeta_{P}(s) \approx \Gamma(-1 / 2) s^{-3 / 2}, \quad \text { as } s \downarrow 0 \text { along } \Im(s)=0 .
$$

On the other hand, by Theorem 8 ,

$$
\operatorname{htr}_{P}(t)=\sum_{n=0}^{\infty} \mathrm{e}^{-t \mathrm{e}^{n^{2 / 3}}} \approx(-\log t)^{3 / 2}, \quad \text { as } t \downarrow 0 .
$$

The above examples show that Tauberian theorems and the inverse Mellin transform have different domains of applicability and can be considered as complementary tools in the study of the asymptotic behaviour of heat traces.

There is also a third method, namely the Poisson summation, not discussed in this paper but widely used in the field of spectral geometry. The Poisson summation formula can be written as

$$
\sum_{n=-\infty}^{\infty} f(t n)=t^{-1} \sum_{k=-\infty}^{\infty} \mathcal{F}[f]\left(\frac{k}{t}\right),
$$

where $\mathcal{F}$ is the Fourier transform and $f$ is a real function satisfying suitable conditions (as usual, the more we assume on $f$, the more we know on the convergence and properties of the Fourier series on the RHS - see [66, 74]). The main advantage of the Poisson summation method is that it allows to obtain the asymptotic expansion of $\operatorname{Tr}(f(t P))$ for quite a general class of functions $f$, whereas the Mellin transform technique in its basic form described in this paper applies only to $f(x)=\mathrm{e}^{-x}$. Hence, Poisson summation method is better suited to spectral action calculations. On the other hand, the Poisson summation may be used only for operators with polynomial spectra (see Section 4.1), and only for those with eigenvalues given by first order 
polynomial. ${ }^{3}$ This is quite restrictive, however many symmetric sphere-like manifolds and their deformations provide Dirac operators with spectra of the desired form [57]. Poisson summation also generalises easily to the multi-index case, which can be applied to obtain spectral action on tori. This cannot be easily done by direct application of Mellin transform technique. ${ }^{4}$ These properties make Poisson summation a convenient method for calculations of spectral action on some specific spaces, but make it less suitable for more general considerations, which are the aim of this paper.

The second distinguishing property of Poisson summation method is the fact, that it provides only the principal part of the spectral action asymptotic expansion. This principal part depends solely on $\mathcal{F}[f](0)$ term in (56). If one is interested in the non-singular part of the expansion, then one needs to analyse the whole RHS of (56). This would arguably neither help in finding the asymptotic expansion, nor say anything about its exactness. We found the Mellin transform technique more useful in achieving the aims of present paper.

Let us now sum up the results of the paper. In Section 3 we have presented general theorems on the existence and convergence of heat traces associated with positive unbounded operators with compact inverses. The necessary conditions were formulated in terms of spectral zeta-functions. The non-existence of meromorphic extensions of the latter sets a natural limitation of applicability of the inverse Mellin transform. However, lacunary operators seem to be pathological anyway from the viewpoint of noncommutative geometry. For instance, if a spectral triple would have a lacunary Dirac operator, then it would not have a dimension spectrum [20]. On the other hand, the assumption (12), even in its more refined version (19), seems to be a mild one. In fact, a similar one was adopted in [20, p. 206] "on the technical side".

In Section 4 devoted to examples we always worked with operators, the spectrum of which is known explicitly. Therefore, the operatorial aspect of the problem was somewhat hidden. In practice, one is rarely granted the comfort of knowing the full spectrum of a given operator. Even if this is the case, one would like study the behaviour of the heat trace when a fixed operator $P$ is perturbed to $P+A$, with some bounded $A$. Clearly, a bounded perturbation of $P$ would not change the leading behaviour of $\operatorname{htr}_{P}(t)$ at small $t$, but it can, at least in principle, spoil the asymptotic expansion.

Indeed, perturbations may drastically change the analytic properties of the associated zeta-functions. For instance, the modulus of the Dirac operator on the standard Podleś sphere has (up to a multiplicative constant) the following eigenvalues $\lambda_{n}\left(\left|\mathcal{D}_{q}\right|\right)=q^{-n}-q^{n}$ (see [27]). It can thus be considered as a sum of an operator of exponential spectrum $P$ and a trace class perturbation $Q$. It turns out that the poles of $\zeta_{P+Q}$ form a regular lattice on the left complex half-plane [29], whereas the poles of $\zeta_{P}$ are located only on the imaginary axis (see Section 4.2). Although, the convergence properties of the small $t$ expansion of $\operatorname{htr}_{P+Q}(t)$ are not altered by

\footnotetext{
${ }^{3}$ One needs to remember, that in Poisson formula eigenvalues are indexed by $\mathbb{Z}$, while in Mellin transform approach the index set is $\mathbb{N}$. Usually this difference can be circumvented, e. g. by using the decomposition (34) which works for both methods.

${ }^{4}$ This is because the spectrum of Dirac operators on tori cannot be easily ordered into an increasing sequence and because the zeta-function would involve multi-index summation.
} 
the perturbation $Q$, but the estimates of contour integrals are much more subtle and tedious to control (see [29, Proposition 4.3]).

We regard the investigation of the impact of perturbations on the asymptotic expansion of heat traces an important and natural next step in the study initiated in this paper. We hope that a combination of our results with the techniques developed in $[7,54,67]$ can lead to a better understanding of heat traces outside the realm of classical pseudodifferential operators.

A promising application of the results presented in the paper concerns the spectral action [15]. By using the semi-group property of heat operators and (distributional) Laplace transform techniques one can attempt to extend both the existence and exactness results to functionals of the form $\operatorname{Tr} f(t P)$ for suitable cut-off functions $f$ see [29] for an explicit example and [28] for a more detailed study. Let us note that the non-perturbative calculations carried out in [10, 57, 58, 61, 68] using Poisson summation formula are, according to the nomenclature adopted in Definition 5, only almost exact. However, with the help of Theorem 5 (compare also [28, Proposition 3.2.2] and [29, Theorem 5.2]) one is actually able to get exact formulae for the spectral action for an explicit class of cut-off functions. This technique may prove potentially useful in the study of cosmic topology [57].

Acknowledgments The authors would like to thank Bruno Iochum and Andrzej Sitarz for numerous valuable discussions.

Project operated within the Foundation for Polish Science IPP Programme "Geometry and Topology in Physical Models" co-financed by the EU European Regional Development Fund, Operational Program Innovative Economy 2007-2013.

Open Access This article is distributed under the terms of the Creative Commons Attribution 4.0 International License (http://creativecommons.org/licenses/by/4.0/), which permits unrestricted use, distribution, and reproduction in any medium, provided you give appropriate credit to the original author(s) and the source, provide a link to the Creative Commons license, and indicate if changes were made.

\section{References}

1. Abramowitz, M., Stegun, I.A.: Handbook of Mathematical Functions: With Formulas, Graphs, and Mathematical Tables. Courier Dover (2012)

2. Avramidi, I.G.: Heat kernel approach in quantum field theory. Nucl. Phys. B, Proc. Suppl. 104(13), 3-32 (2002). doi:10.1016/S0920-5632(01)01593-6. Proceedings of the international meeting on quantum gravity and spectral geometry

3. Bär, C.: The Dirac operator on space forms of positive curvature. Journal of the Mathematical Society of Japan 48(1), 69-83 (1996). doi:10.2969/jmsj/04810069

4. Bär, C.: Dependence of the Dirac spectrum on the spin structure. Séminaires et Congrés 4, 1733 (2000). Global Analysis and Harmonic Analysis, Jean Pierre Bourguignon - Thomas Branson - Oussama Hijazi (Ed.) http://www.emis.ams.org/journals/SC/2000/4/html/smf_sem-cong_4_17-33. html

5. Bertlmann, R.A.: Anomalies in Quantum Field Theory. International Series of Monographs on Physics, vol. 91. Clarendon (1996)

6. Camporesi, R., Higuchi, A.: On the eigenfunctions of the Dirac operator on spheres and real hyperbolic spaces. J. Geom. Phys. 20(1), 1-18 (1996). doi:10.1016/0393-0440(95)00042-9

7. Carey, A., Sukochev, F.: Measurable operators and the asymptotics of heat kernels and zeta functions. J. Funct. Anal. 262(10), 4582-4599 (2012). doi:10.1016/j.jfa.2012.03.008 
8. Carey, A.L., Phillips, J., Rennie, A., Sukochev, F.A.: The local index formula in semifinite von Neumann algebras I: spectral flow. Adv. Math. 202(2), 451-516 (2006). doi:10.1016/j.aim.2005.03.011

9. Chamseddine, A.H., Connes, A.: The spectral action principle. Commun. Math. Phys. 186(3), 731750 (1997). doi:10.1007/s002200050126

10. Chamseddine, A.H., Connes, A.: Spectral action for Robertson-Walker metrics. Commun. Math. Phys. 1210, 101 (2012). doi:10.1007/JHEP10(2012)101

11. Christensen, E., Ivan, C., Lapidus, M.L.: Dirac operators and spectral triples for some fractal sets built on curves. Adv. Math. 217(1), 42-78 (2008). doi:10.1016/j.aim.2007.06.009

12. Christensen, E., Ivan, C., Schrohe, E.: Spectral triples and the geometry of fractals. Journal of Noncommutative Geometry 6(2), 249-274 (2012). doi:10.4171/JNCG/91

13. Cipriani, F., Guido, D., Isola, T., Sauvageot, J.L.: Spectral triples for the Sierpiński gasket. J. Funct. Anal. 266(8), 4809-4869 (2014). doi:10.1016/j.jfa.2014.02.013

14. Comtet, L.: Advanced combinatorics. The Art of Finite and Infinite Expansions. doi:10.1007/978-94-010-2196-8. Springer, Berlin (1974)

15. Connes, A.: The action functional in non-commutative geometry. Commun. Math. Phys. 117(4), 673683 (1988)

16. Connes, A.: Noncommutative Geometry. Academic, New York (1995)

17. Connes, A.: Cyclic cohomology, quantum group symmetries and the local index formula for $S U_{q}(2)$. Journal of the Institute of Mathematics of Jussieu 3(1), 17-68 (2004). doi:10.1017/S1474748004000027

18. Connes, A., Landi, G.: Noncommutative manifolds, the instanton algebra and isospectral deformations. Commun. Math. Phys. 221(1), 141-159 (2001). doi:10.1007/PL00005571

19. Connes, A., Marcolli, M.: Noncommutative Geometry, Quantum Fields and Motives, Colloquium Publications, vol. 55. American Mathematical Society (2008)

20. Connes, A., Moscovici, H.: The local index formula in noncommutative geometry. Geom. Funct. Anal. GAFA 5(2), 174-243 (1995). doi:10.1007/BF01895667

21. Connes, A., Moscovici, H.: Modular curvature for noncommutative two-tori. J. Am. Math. Soc. 27(3), 639-684 (2014). doi:10.1090/S0894-0347-2014-00793-1

22. Copson, E.T.: Asymptotic expansions. In: No. 55 in Cambridge Tracts in Mathematics. Cambridge University Press, Cambridge (1965)

23. Costin, O., Huang, M.: Behavior of lacunary series at the natural boundary. Adv. Math. 222(4), 13701404 (2009). doi:10.1016/j.aim.2009.06.011

24. Dąbrowski, L., D’Andrea, F., Landi, G., Wagner, E.: Dirac operators on all Podlés quantum spheres. Journal of Noncommutative Geometry 1(2), 213-239 (2007). doi:10.4171/JNCG/5

25. Dąbrowski, L., Landi, G., Paschke, M., Sitarz, A.: The spectral geometry of the equatorial Podleś sphere. Comptes Rendus Mathematique 340(11), 819-822 (2005). doi:10.1016/j.crma.2005.04.003

26. Dąbrowski, L., Landi, G., Sitarz, A., Suijlekom, W.V., Várilly, J.C.: The Dirac operator on $S U_{q}(2)$. Commun. Math. Phys. 259(3), 729-759 (2005). doi:10.1007/s00220-005-1383-9

27. Dąbrowski, L., Sitarz, A.: Dirac operator on the standard Podleś quantum sphere. Banach Center Publications 61, 49-58 (2003). doi:10.4064/bc61-0-4

28. Eckstein, M.: Spectral action - beyond the almost commutative geometry. Ph.D. thesis, Jagiellonian University (2014)

29. Eckstein, M., Iochum, B., Sitarz, A.: Heat trace and spectral action on the standard Podleś sphere. Commun. Math. Phys. 332(2), 627-668 (2014). doi:10.1007/s00220-014-2054-5

30. Elizalde, E., Romeo, A.: Rigorous extension of the proof of zeta-function regularization. Phys. Rev. D 40(2), 436-443 (1989). doi:10.1103/PhysRevD.40.436

31. Erdélyi, A.: Asymptotic expansions courier. Dover, New York (1956)

32. Essouabri, D., Iochum, B., Levy, C., Sitarz, A.: Spectral action on noncommutative torus. Journal of Noncommutative Geometry 2(1), 53-123 (2008). doi:10.4171/JNCG/16

33. Fabry, E.: Sur les séries de taylor qui ont une infinité de points singuliers. Acta Math. 22(1), 65-87 (1899). doi:10.1007/BF02417871

34. Feller, W.: An Introduction to Probability Theory and its Applications, vol. 2. Wiley, New York (2008)

35. Flajolet, P., Gourdon, X., Dumas, P.: Mellin transforms and asymptotics: harmonic sums. Theor. Comput. Sci. 144(1), 3-58 (1995). doi:10.1016/0304-3975(95)00002-E

36. Fulling, S.A.: Aspects of Quantum Field Theory in Curved Spacetime. London Mathematical Society Student Texts, vol. 17. Cambridge University Press, Cambridge (1989) 
37. Gayral, V., Iochum, B., Vassilevich, D.: Heat kernel and number theory on NC-torus. Commun. Math. Phys. 273(2), 415-443 (2007). doi:10.1007/s00220-007-0194-6

38. Gayral, V., Wulkenhaar, R.: Spectral geometry of the Moyal plane with harmonic propagation. Journal of Noncommutative Geometry 7(4), 939-979 (2013). doi:10.4171/JNCG/140

39. Gilkey, P.B., Toledo, D.: Invariance Theory, the Heat Equation, and the Atiyah-Singer Index Theorem, 2nd edn. Studies in Advanced Mathematics. CRC Press, Boca Raton (1998)

40. Gilkey, P.B., Toledo, D.: Invariance Theory, the Heat Equation, and the Atiyah-Singer Index Theorem, 2nd edn. Studies in Advanced Mathematics. CRC Press, Boca Raton (1995)

41. Guido, D., Isola, T.: Dimensions and singular traces for spectral triples, with applications to fractals. J. Funct. Anal. 203(2), 362-400 (2003). doi:10.1016/S0022-1236(03)00230-1

42. Guido, D., Isola, T.: Dimensions and spectral triples for fractals in $\mathbb{R}^{n}$. In: Boca, F., Bratteli, O., Longo, R., Siedentop, H. (eds.) Advances in Operator Algebras and Mathematical Physics, Theta Series in Advanced Mathematics, pp. 89-108 (2005)

43. Hardy, G.H. Divergent Series, 2nd edn. American Mathematical Society (1991)

44. Hardy, G.H., Riesz, M.: The General Theory of Dirichlet's Series. Cambridge Tracts in Mathematics and Mathematical Physics, vol. 18. Cambridge University Press, Cambridge (1915)

45. Hirschman, I.I., Jenkins, J.A.: On lacunary Dirichlet series. Proc. Am. Math. Soc. 1(4), 512-517 (1950). doi:10.2307/2032321

46. Iochum, B., Levy, C., Vassilevich, D.: Global and local aspects of spectral actions. J. Phys. A Math. Theor. 45(37), 374,020 (2012). doi:10.1088/1751-8113/45/37/374020

47. Iochum, B., Levy, C., Vassilevich, D.: Spectral action beyond the weak-field approximation. Commun. Math. Phys. 316(3), 595-613 (2012). doi:10.1007/s00220-012-1587-8

48. Iochum, B., Levy, C., Vassilevich, D.: Spectral action for torsion with and without boundaries. Commun. Math. Phys. 310(2), 367-382 (2012). doi:10.1007/s00220-011-1406-7

49. Jonquiére, A.: Note sur la série $\sum_{n=1}^{\infty} \frac{x^{n}}{n^{s}}$. Bulletin de la Société Mathématique de France 17, 142-152 (1889). http://eudml.org/doc/85669

50. Kaad, J., Senior, R.: A twisted spectral triple for quantum $S U(2)$. J. Geom. Phys. 62(4), 731-739 (2012). doi:10.1016/j.geomphys.2011.12.019

51. Kakehi, T., Masuda, T.: Logarithmic divergence of heat kernels on some quantum spaces. Tôhoku Mathematical Journal 47(4), 595-600 (1995). doi:10.2748/tmj/1178225463

52. Kaneko, M., Kurokawa, N., Wakayama, M.: A variation of Euler's approach to values of the Riemann zeta function. Kyushu Journal of Mathematics 57(1), 175-192 (2003). doi:10.2206/kyushujm.57.175

53. Lesch, M.: On the noncommutative residue for pseudodifferential operators with log-polyhomogeneous symbols. Ann. Glob. Anal. Geom. 17(2), 151-187 (1999). doi:10.1023/A:1006504318696

54. Lord, S., Sukochev, F., Zanin, D.: Singular Traces: Theory and Applications. De Gruyter Studies in Mathematics, vol. 46. Walter de Gruyter (2012)

55. Mandelbrojt, S.: Séries lacunaires. Actualités Scientifiques Et Industrielles, p. 305, Paris (1936)

56. Mandelbrojt, S., Miles, E.R.: Lacunary functions. The Rice Institute Pamphlet 14(4), 261-284 (1927). http://hdl.handle.net/1911/8511

57. Marcolli, M., Pierpaoli, E., Teh, K.: The spectral action and cosmic topology. Commun. Math. Phys. 304(1), 125-174 (2011). doi:10.1007/s00220-011-1211-3

58. Marcolli, M., Pierpaoli, E., Teh, K.: The coupling of topology and inflation in noncommutative cosmology. Commun. Math. Phys. 309(2), 341-369 (2012). doi:10.1007/s00220-011-1352-4

59. Matsumoto, K., Weng, L.: Zeta-functions defined by two polynomials. In: Number Theoretic Methods. doi:10.1007/978-1-4757-3675-5_13, pp. 233-262. Springer, Berlin (2002)

60. Neshveyev, S., Tuset, L.: A local index formula for the quantum sphere. Commun. Math. Phys. 254(2), 323-341 (2005). doi:10.1007/s00220-004-1154-Z

61. Olczykowski, P., Sitarz, A.: On spectral action over Bieberbach manifolds. Acta Phys. Pol. B 42(6), 1189-1198 (2011). doi:10.5506/APhysPolB.42.1189

62. Pal, A., Sundar, S.: Regularity and dimension spectrum of the equivariant spectral triple for the odd-dimensional quantum spheres. Journal of Noncommutative Geometry 4(3), 389-439 (2010). doi:10.4171/JNCG/61

63. Paris, R.B., Kaminski, D.: Asymptotics and Mellin-Barnes Integrals. Encyclopedia of Mathematics and its Applications, vol. 85. Cambridge University Press, Cambridge (2001)

64. Schrohe, E.: Complex powers of elliptic pseudodifferential operators. Integr. Equ. Oper. Theory 9(3), 337-354 (1986). doi:10.1007/BF01199350

65. Shubin, M.A.: Pseudodifferential Operators and Spectral Theory. Springer, Berlin (2001) 
66. Stein, E., Weiss, G.L.: Introduction to Fourier analysis on Euclidean spaces. Princeton University Press, Princeton (1971)

67. Sukochev, F., Zanin, D.: $\zeta$-function and heat kernel formulae. J. Funct. Anal. 260(8), 2451-2482 (2011). doi:10.1016/j.jfa.2010.10.007

68. Teh, K.: Dirac spectra, summation formulae, and the spectral action. Ph.D. thesis, California Institute of Technology. http://resolver.caltech.edu/CaltechTHESIS:05082013-134706988 (2013)

69. Trautman, A.: Spin structures on hypersurfaces and the spectrum of the Dirac operator on spheres. In: Spinors, twistors, Clifford algebras and quantum deformations. doi:10.1007/978-94-011-1719-7_3, pp. 25-29. Springer, Berlin (1993)

70. Vassilevich, D.V.: Heat kernel expansion: user's manual. Phys. Rep. 388(5), 279-360 (2003). doi:10.1016/j.physrep.2003.09.002

71. Vassilevich, D.V.: Noncommutative heat kernel. Lett. Math. Phys. 67(3), 185-194 (2004). doi:10.1023/B:MATH.0000035037.50663.b1

72. Vassilevich, D.V.: Heat trace asymptotics on noncommutative spaces. SIGMA 3(093), 0708-4209 (2007). doi:10.3842/SIGMA.2007.093

73. Widder, D.V.: The Laplace Transform. Princeton University Press, Princeton (1946)

74. Zygmund, A.: Trigonometric series, 2nd edn., repr. with corrections and some addictions edn. Cambridge University Press, Cambridge (1968) 